\title{
THE RIGHT TIME, THE RIGHT PLACE
}

An expert examination of the application of health and social care governance arrangements for ensuring the quality of care provision in Northern Ireland

DECEMBER 2014

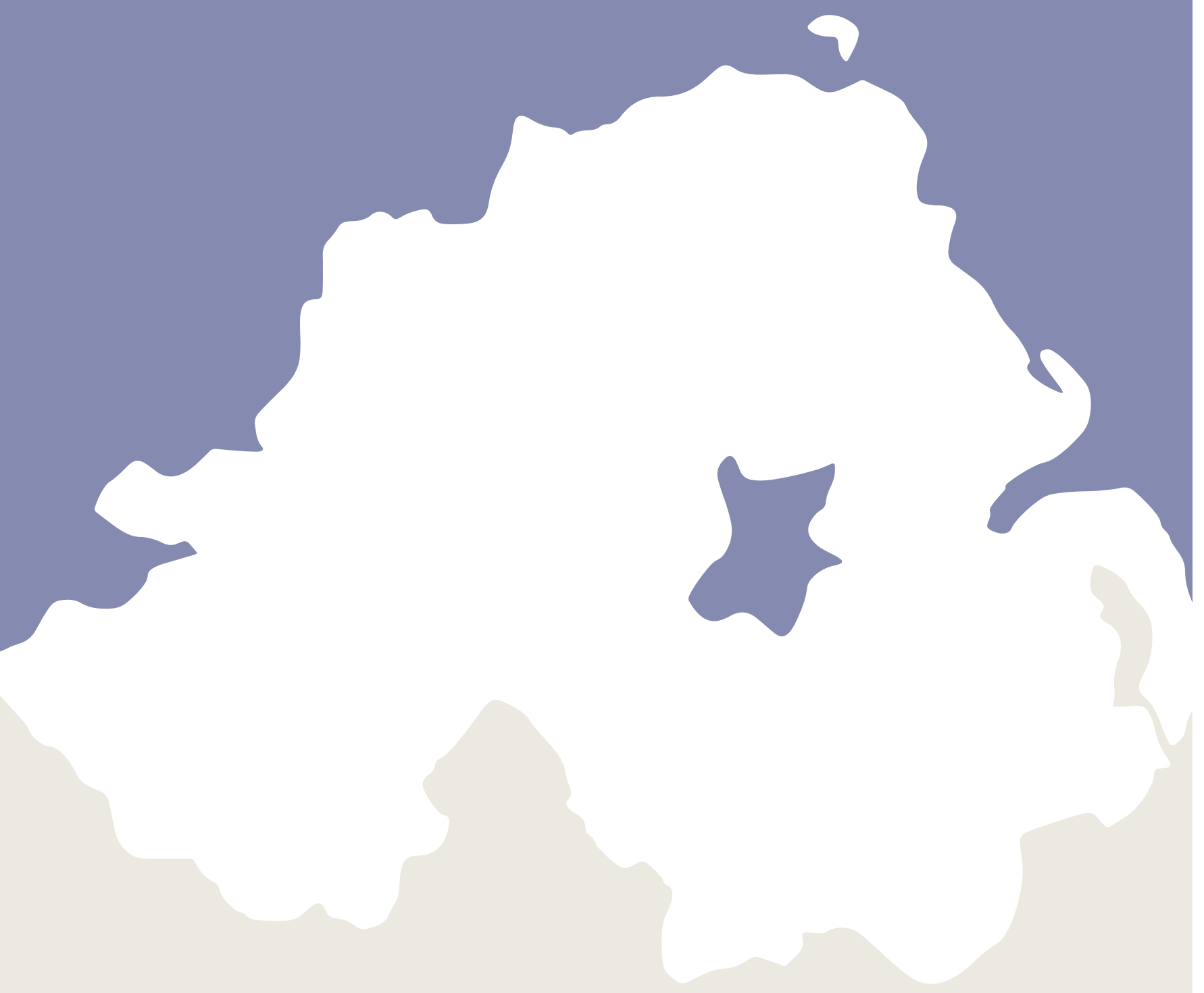

Review Team | Sir Liam Donaldson | Dr Paul Rutter | Dr Michael Henderson 


\section{CONTENTS}

1 CONTEXT

4.1 A system under the microscope 8

4.2 The design of the system hinders high quality, safe care $\quad 11$

4.3 Insufficient focus on the key ingredients of quality and safety improvement 18

4.4 Extracting full value from incidents and complaints 22

4.5 The benefits and challenges of being open 34

4.6 The voices of patients, clients and families are too muted 37

5 CONCLUSIONS $\quad 39$

5.1 Relative safety of the Northern Ireland care system 39

5.2 Problems generated by the design of the health and social care system 39

5.3 Focus on quality and safety improvement $\quad 40$

5.4 The extent to which Serious Adverse Incident reporting improves safety 41

5.5 Openness with patients and families 43

$\begin{array}{lll}6 & \text { RECOMMENDATIONS } & 44\end{array}$ 


\section{CONTEXT}

Throughout the developed world much healthcare is of a very high standard. The range of technologies and drugs available to diagnose and treat illness greatly increased during the second half of the 20th Century, and into the 21 st, offering life and hope where patients' prospects were once bleak. As a consequence, the number of people living with disease and needing years or even decades of support from care systems has expanded enormously.

The ageing population of today is a central consideration in a way that was not foreseen when modern healthcare came into being in the aftermath of the Second World War. Today, people are living much longer and developing not just one disease but several that co-exist. In old age, the twin states of multi-morbidity and frailty are creating acute and long-term health and social care needs on an unprecedented scale.

Technology has continued its rapid and beneficial advance, opening up new opportunities for diagnosis and treatment but bringing even greater numbers through the doors of hospitals and health centres. Citizens experience the benefits of an advanced consumer society and when they encounter the health and social care system, they rightly expect it to be commensurate with this. Rising public expectations are a further driver of demand for healthcare. There are other, less predictable sources of pressure on services. For example, a change in the pattern of winter viruses can bring surges in demand that threaten to overwhelm emergency departments. In response to all of this, the size of budgets devoted to health and social care has had to expand dramatically.

At the epicentre of this complex, pressurised, fast-moving environment is the patient. The primary goal of the care provided must always be to make their experience, the outcome of their condition, their treatment, and their safety as good as it gets. Health and social care systems around the world struggle to meet this simple ideal. Evaluations repeatedly show that: variation in standards of care within countries is extensive; some of the basics such as cleanliness and infection are too often neglected; evidence-based best practice is adopted slowly and inconsistently; the avoidable risks of care are too high; there are periodic instances of serious failures in standards of care; and, many patients experience disrespect for them and their families, bad communication and poor coordination of care.

The health and social care system in Northern Ireland serves a population of 1.8 million. People live in urban, semi-rural or rural communities. Responsibility for population health and wellbeing, and the provision of health and social care, is devolved to the Northern Ireland Assembly from the United Kingdom government in Westminster. As in other parts of the United Kingdom, the Northern Ireland health service operates based on the founding principles of the National Health Service - the provision of care according to need, free at the point of access and beyond, funded from taxation. However, since the advent of devolved government, England, Scotland, Wales and Northern Ireland have adopted their own strategies for: promoting and protecting health; preventing disease; reducing health inequalities; and, planning and providing health and social care services. The countries have developed different structures and functions within their systems to meet these responsibilities. Thus, they vary in features such as: arrangements for planning and contracting of care; levels of investment in public health, primary and community care versus hospital provision; funding models; incentives; use of the independent sector; managerial structures; and, the role of the headquarters function.

Various agencies, groups and strategies populate the quality and safety landscape of Northern Ireland. Quality 2020 is the flagship 
ten-year strategy. Commissioned by the Minister of Health, Social Services and Public Safety in 2011, its vision is to make Northern Ireland an international leader in high quality, safe care. Quality 2020 is sponsored by the Chief Medical Officer and led by the Department of Health, Social Services and Public Safety. It has a steering group, a management group, an implementation team, project teams, and a stakeholder forum. These bring together representatives from across the statutory care bodies and beyond. Separately, a Health and Social Care Safety Forum convenes a similar group of stakeholders.

The Regulation and Quality Improvement Authority (RQIA) is the main regulator in Northern Ireland's care system. Many of the social care providers, and some healthcare providers, are registered with the Regulation and Quality Improvement Authority. However it does not register the Trusts, which provide the bulk of health and social care in Northern Ireland, or general practices. The Trusts' relationship with the regulator therefore has a somewhat softer edge than might be the case if they were formally registered, although an expanded role has been announced recently by the Minister.

Northern Ireland takes a keen interest in the work of quality and safety bodies elsewhere in the United Kingdom, and often implements their guidance and recommendations.

The National Institute for Health and Care Excellence (NICE) and the former National Patient Safety Agency have been prominent in this regard.

Technical quality and safety expertise sits not in the Health and Social Care Board, but next door in the Public Health Agency. The Public Health Agency has a statutory role in approving the Health and Social Care Board's commissioning plans. Two executive directors are jointly appointed between the Public Health
Agency and the Health and Social Care Board. There are therefore mechanisms through which quality and safety expertise should inform the Board's work. The Quality Safety Experience Group is jointly managed between these two agencies. It meets monthly and its primary focus is learning. It looks at patterns and trends in incidents and initiates thematic reviews.

In short, there is a good degree of activity in the sphere of quality and safety improvement. There are some unusual features of the landscape, which will emerge in some detail in this Review.

The way in which central bodies seek to achieve compliance with their policies and make broader improvement changes is based on a very traditional and quite bureaucratic management model. There is much detailed specification of what to do, how to do it, and then extensive and detailed checking of whether it has been done. This has strengths in enabling the central bodies and the government to demonstrate their accountability and give public assurances, but it can greatly disempower those at the local level. It can cause those managing locally to look up, rather than looking out to the needs of their populations.

The alternative is a style of leadership based on inspiration, motivation and trust that those closer to the front line will make good judgments and innovate if they are encouraged to do so. Perhaps the relationship needs a lighter touch, to liberate freer thinking on how to make services better for the future. 


\section{TERMS OF REFERENCE} AND WORKING METHODS

The Review's formal Terms of Reference are available online ${ }^{1}$. The overall aim of the Review has been to examine the arrangements for assuring and improving the quality and safety of care in Northern Ireland, to assess their strengths and weaknesses, and to make proposals to strengthen them.

The analysis in this report is based on extensive input from, scrutiny of, and discussion with people across the health and social care system in Northern Ireland. Each of the main statutory organisations made formal submissions to the Review (including records of board meetings, policies, and plans). The Review put substantial emphasis on travelling around the system both literally and figuratively - to see it from as many different angles as possible, and to come to a rounded view.

The Review Team visited the five Health and Social Care Trusts, the Northern Ireland Ambulance Service, the Department of Health, Social Services and Public Safety, the Health and Social Care Board land its Local Commissioning Groups), the Public Health Agency, the Patient and Client Council, and the Regulation and Quality Improvement Authority. In each, the Review Team met with the executive team (Chief Executive and executive directors) and, in most cases, the Chair of the Board and other non-executive directors. The management team of each organisation gave a series of presentations covering the areas of interest to the Review, and Review Team members asked questions and led discussion.

During their visit to each Health and Social Care Trust and to the ambulance service, Review Team members also led focus groups discussions amongst frontline staff. In each of the five Health and Social Care Trusts, for example, the team met with separate groups of consultants, nurses, junior doctors, and other health and social care professionals. Senior managers were not present for these discussions. Participants were encouraged to speak openly, and generally did so. It was understood that no comments would be attributed to individuals. The focus groups centered on any concerns about quality and patient safety in their organisation and incident reporting, and other highly-related topics. The team also met with two groups of general practitioners.

The Review Team paid particular attention to the experiences of people who have come to harm within the Northern Ireland health and social care system. At each Trust, including the ambulance service, the team reviewed two recent Serious Adverse Incidents in detail, particularly considering the incident itself, the way in which patients and families were kept informed and involved, and the learning derived. The team later returned to two Trusts to review further incidents, this time selected by the Review Team from a list of all serious adverse incidents in the previous year. The Review Team met with people who have come to harm. Most of these meetings were in person; some were by telephone. In addition to people affected directly, the Review Team spoke to their family members and carers. We are particularly grateful to all of these individuals for giving of their time, and for graciously sharing their stories with us, which were often painful.

Finally, the Review Team met with a series of other individuals and groups that form part of the wider health and social care system in Northern Ireland, or have a strong interest in it. These were: the Attorney General, the British Medical Association, the Chest Heart and Stroke Association, the Commissioner for Older People for Northern Ireland, Diabetes UK, the General Medical Council, MacMillan Cancer Support, the Multiple Sclerosis Society, the Northern Ireland Association of Social Workers, the Northern Ireland Human Rights Commissioner, the Northern Ireland Medical \& Dental Training Agency, The Honourable Mr Justice O'Hara, 
the Ombudsman for Northern Ireland, the Pain Alliance of Northern Ireland, Patients First Northern Ireland, the Royal College of Nursing, and the Voice of Young People in Care. Other patient and client representative groups were invited to meet with the Review Team, or to make written submissions.

To inform one aspect of the Review, the Regulation and Quality Improvement Authority oversaw a look-back exercise, reviewing the handling of all Serious Adverse Incidents in Northern Ireland between 2009 and 2013. Their report was received late in the Review process, but has been considered by the Review Team and reflected in this report.

Between starting and producing its final report, the Review Team has had a relatively short period of time. It has not been possible to undertake research, extensive data analysis, large-scale surveys of opinion, or formal evidence-taking sessions. However, the documents reviewed, the meetings held, the visits made, and the views heard have given a strikingly consistent picture of quality and safety in the Northern Ireland health and social care system. The Review Team is confident that a longer exercise would not have produced very different findings. 


\section{THE CHALLENGES OF DELIVERING HIGH QUALITY, SAFE CARE}

Patients in hospitals and other health and social care services around the world die unnecessarily, and are avoidably injured and disabled. This sad fact has become well known since the turn of the 20th Century. Awareness of it has not been matched, unfortunately, by effective action to tackle it.

There is consistency in the types of harm that occur in high-income countries. In low-income countries, harm is mainly related to lack of infrastructure and facilities, as well as poor access to care. However, in North America, Europe, Australasia, and many parts of Asia and the Middle East, analysis of incident reports and the findings of patient safety research studies shows a different, strikingly consistent pattern. Between $3 \%$ and $25 \%$ of all hospital admissions result in an adverse incident, about half potentially avoidable. Within any health or social care service, there are many potential threats to the quality and safety of the care provided:

1. Weak infrastructure - the range and distribution of facilities, equipment and staff is inadequate to provide fair and timely access to required care.

2. Poor co-ordination - the components of care necessary to meet the needs of a patient, or group of patients, do not work well together to produce an effective outcome and to be convenient to patients and their families.

3. Low resilience - the defences in place, and the design of processes of care, are insufficient to reliably protect against harm such as that resulting from errors or from faulty and misused equipment.

4. Poor leadership and adverse culture - the organisation or service providing care does not have clear goals and a philosophy of care that it is embedded in the values of the organisation and visible in every operational activity.

5. Competence, attitudes, and behaviour - the practitioners and care-providers working within the service lack the appropriate skills to deal with the patients that they encounter, or they are unprofessional in their outlook and actions, or they do not respect other team members, nor work effectively with them.

6. Sub-optimal service performance - the way that the service is designed, organised and delivered means that it does not deliver processes of care to a consistently high standard so that over time it chronically under-performs often in a way that is not noticed until comparative performance is looked at.

7. Slow adoption of evidence-based practice - the service does not conform to international best practice in particular areas of care or overall.

The amount of each type of harm varies but the overall burden has changed little over the last decade despite the unprecedented priority that has been given to patient safety within these health systems. Little is known about the level and nature of harm in primary care, though more attention is now being given to it.

Although these threats are described in relation to health, they apply also to social care. Many are strongly related to the level of resources that is available to a health and social care system. The extent to which each problem is present varies hugely across the world, within countries, and even between different parts of the same service or area of care provision.

In some ways it is reassuring to believe that the problems of quality and safety of care are somehow universal, and that no country has the answers. This is dangerous thinking. The best services in the world show that even with the all the pressures of large numbers of patients, many with complex needs, excellence can be achieved consistently across all fields of care. The Northern Ireland health and social care service must not be satisfied with "good enough.' With a clear recognition of the reasons for its current problems in quality and safety of care, and with everyone working together, it could be amongst the best in the world. 


\section{KEY THEMES ESTABLISHED BY THE REVIEW}

The Review established six key themes. Each is set out in some detail below. Exploration of these themes provides the basis for the Review's conclusions (in section 5) and recommendations (section 6).

\subsection{A SYSTEM UNDER THE MICROSCOPE}

Northern Ireland's health and social care system is subject to a high, perhaps unrivalled, level of media coverage - much of it negative. Over recent years, it has also been the subject of a series of high profile inquiries. All have highlighted numerous failings in the leadership and governance of care. Many have made extensive recommendations and the extent to which these have been implemented has itself been controversial. The pressures of increasing demand for care have meant that access has been more difficult. There has been a focus on over-crowding and delays in emergency departments, the front door of the hospital service. All of this has meant that the last five years has been a period of unprecedented scrutiny of the way that health and social care in Northern Ireland is planned, provided and funded.

\subsubsection{A stream of inquiries highlighting service failures}

The number of recent major investigations and inquiries into shortfalls in standards of care in health and social care services in Northern Ireland is striking in relation to the size of its population. This does not necessarily mean that such occurrences are commoner than elsewhere in the United Kingdom. It may simply be that the level of public and media scrutiny is higher and the pressure from this triggers a statutory response by government ministers and officials. The end-result is that the profile of the service is more often one of failure rather than success.
In March 2011, Dame Deirdre Hine, a former Chief Medical Officer for Wales, issued the report of her inquiry into deaths from Clostridium difficile in hospitals in the Northern Trust area. She had been brought in to investigate 60 deaths that had been attributed to the organism. She found that the true figure was 31 deaths. She found management, organisational, clinical governance and communication failings. She made 12 recommendations. It took 23 months to complete.

In February 2011, the Belfast Trust recalled 117 dental patients following a review of the clinical performance of a senior consultant. An independent inquiry commissioned by the Minister was published in July 2013 and made 45 recommendations. An action plan developed by the Department of Health, Social Services and Public Safety identified 42 key actions including on staffing, training, supervision and clinical governance. In November 2013, the Regulation and Quality Improvement Authority conducted an assessment of implementation of those actions.

In December 2011, an independent report by the Regulation and Quality Improvement Authority examined delays in the reporting of plain X-rays in all Trusts after concerns were expressed about delays in two hospitals. The review found that serious delays had occurred and were caused by three main factors: a shortfall in consultant radiology staffing, a growth in numbers of $x$-rays to be reported after the introduction of digital imaging and the introduction of a new policy to report on all hospital chest $x$-rays because of worries about patient safety. The review found that there was little awareness at regional level that a serious backlog in reporting was developing with potential risks to patients due to delayed diagnosis. The review made 14 recommendations. 
In May 2012, Doctor Pat Troop, former chief executive officer of the Health Protection Agency in England, issued her final report of the independent investigation into an outbreak of infections in neonatal units due to the organism Pseudomonas aeruginosa. Five babies had died in the outbreak and 32 recommendations were made covering technical matters, management, governance, communication, training, and outbreak management.

In April 2012, the Minister asked for special measures to be put in place to oversee the Belfast Trust because of major concerns about serious adverse incidents in the emergency department, recommendations from the Pseudomonas review, reviews of paediatric congenital cardiac surgery and recommendations of the dental inquiry.

In December 2012, the Minister appointed a Turnround and Support Team to go into the Northern Health and Social Care Trust because of concerns about the weakness of governance and quality assurance systems, the paucity of clinical leadership, and uncertainties about the reliability of mortality data. This particular Trust has had five chief executive officers in the last seven years.

In June 2014, the Regulation and Quality Improvement Authority reported on its review of unscheduled care services in the Belfast Trust. The concerns that led to the review included: the declaration of a major incident, 12-hour waiting time breaches, dysfunctional patient flows and gross overcrowding of patient care areas. This triggered a fuller review that looked at matters region-wide. This produced 16 recommendations.

The dominant inquiry in recent times remains the Independent Inquiry into HyponatraemiaRelated Deaths. It is examining the deaths of children after being transfused in hospital with a fluid that was subsequently found to carry a significant risk. Concerns had been raised by the parents and others that this risk should have been identified much earlier, that action should have been taken to stop it being used, that there was a cover-up and that systems for monitoring safety were inadequate. It is being chaired by John O'Hara QC and was commissioned in 2003/4 but, because of other legal processes, was not able to hear full evidence until more recently. The report is expected in 2015.

The criticisms in inquiries like these have been largely justified and must be followed by action to improve the situations. Whether establishing formal, often lengthy, and costly inquiries is the right way to drive improvement is very debatable. Certainly doing so as the normative response to failure has important disadvantages. In particular, it often paralyses the organisation under scrutiny as its staff become pre-occupied with preparing evidence and supplying information. The learning is often put on hold - sometimes never to be returned to - until the inquiry is over. The burden of recommendations to be implemented and progress-checked can be overwhelming, so that the implementation becomes a bureaucratic exercise rather than a watershed moment for leadership, culture and the content of practice. It might be better to define a clear threshold for when a full-blown inquiry is initiated.

\subsubsection{Intense political and media interest in service provision}

Northern Ireland's health and social care system is subject to a high degree of political, as well as media, interest. This is a valid and expected feature of a publicly-funded system. Ironically, though, the way in which this interest becomes manifest often creates results that are counter to the true public interest. There have been many examples of local communities - and therefore their politicians - wanting to keep a local hospital open, contrary to the analysis of service planners. This has created 
a situation in which Northern Ireland has more inpatient units than is really justified for the size of population, and the expense of maintaining them impedes provision of other services that would represent better value for money and more appropriately meet the needs of the population. Likewise, political pressure and media interest has prevented the salaries of top managers from being raised too substantially. However, senior executives in the Northern Ireland care system are now paid much less than their counterparts elsewhere in the United Kingdom. The public would be better served if their care system could compete to attract the very best managerial talent. The pressure to keep salaries down may be penny-wise and pound-foolish. 


\subsection{THE DESIGN OF THE SYSTEM HINDERS HIGH QUALITY, SAFE CARE}

When a quality or safety problem arises somewhere within the Northern Ireland care system, the tendency is to point to the individuals or services involved, and to find fault there. As with so many other features identified in this report, this tendency is far from unique to Northern Ireland. But it represents, in the view of the Review Team, too narrow a focus. In reality, the greatest threats to the quality of care that patients receive, and to their safety, come from the way in which the system as a whole is designed and operates.

In short, the services that exist are not the services that the population truly requires. Political and media pressure acts to resist change, despite the fact that change is much needed. It is not clear who is in charge of the system, and the commissioning system is underpowered. All of this compounds the pressures, creating high intensity environments that are stressful for staff and unsafe for patients - particularly out of hours. These effects are explored further below.

The Northern Ireland care system has some elements in common with the other United Kingdom countries, and some that differ. Observers, asked to describe the Northern Ireland system, often point first to the integration of health and social care as its distinguishing feature. It is clear though from the findings of this Review that whilst the integrated design of the system has great advantages, it falls well short of perfection in promoting the highest standards of care and in preventing the dysfunctions in the co-ordination of care that are prevalent elsewhere.

\subsubsection{Service configuration creates safety concerns}

A striking feature of the provision of care in Northern Ireland is the wide distribution of hospital-type facilities outside the major city, Belfast, some serving relatively small populations by United Kingdom standards. This geographical pattern leads to specialist expertise being too thinly spread, and to the patchy availability of experienced and fully competent staff. It means that it is not possible everywhere to deliver the same quality of service for an acutely ill person at 4 a.m. on a Sunday as at $40^{\prime}$ clock on a Wednesday afternoon. There is therefore a two-tier service operating in Northern Ireland - in-hours and out-of-hours - that is more pronounced in some places than in others. This is one of the biggest influences on the quality and safety of care. Delivery of services is too often higher risk than it should be in a 21 st Century healthcare system because of the pattern of services.

Past analysts and observers have pointed to the current level and siting of provision not being in keeping with maintaining high standards of care. Some populations are just too small to warrant full-blown general hospital facilities yet they are kept in place because of public and political pressure. Amongst those who work within the system, there is deep frustration that the public are not properly informed about the higher risks of smaller hospitals and that the misapprehension that alternative forms of provision are in some way inferior to a hospital. These issues are illuminated by two wry comments made to the Review: "the word 'hospital' should be removed from the Oxford English Dictionary" and "Northern Ireland needs more roads not more hospitals."

Despite its small size, there is less co-operative working across Northern Ireland than might be expected. Silos reign supreme. The Health and Social Care Board runs regional commissioning teams, covering areas such as learning 
disability, mental health, prison health and a very broad category of "hospital and related services'. However, particular scope exists to do more in improving standards in areas of clinical care where there is a strong evidence base for what is effective. In the cases where clinicians have worked together across organisational boundaries, remarkable transformations have occurred. This happened in cardiology where a regionally planned and coordinated service means that more patients with heart attacks get treated early, get less damage to their hearts, and more people live rather than die. The Ambulance Trust is the only one of the six Trusts organised on a regional basis. The Review Team was very struck by how much pressure this important service was under. This is consistent with the headline stories in other parts of the United Kingdom about ambulance services being unable to meet their service standards because of huge surges in demand. All parts of the service are taking the strain from those in the control centre to those on the road. Yet when the detail of their situations is explored in depth, it is clear again that the problems stem from dysfunctional patient flows and pathways where different parts of the system are not working together.

\subsubsection{Adverse consequences for primary and social care}

The pressures on hospitals have consequences for primary and community services. There is a constant need for hospitals to discharge patients as soon as they possibly can to free-up beds for new admissions. Generally, this happens when an older person is judged medically fit for discharge. However, this does not necessarily mean that their physical and social functioning has reached a level where they can cope with a return to the community. The Review was told by general practitioners and social care staff that they often have to step in to provide unscheduled support in such circumstances and, because of inadequate communication at the time of discharge, they can be left in the dark about ongoing treatment plans and even be unclear about something as basic as a patient's medication regime. Some general practitioners spoke of spending long, frustrating hours trying to get to speak to a hospital doctor about their patient, without success.

Over the last decade, there has been a major increase in the dependency levels of people being cared for in the community. For example, the use of PEG feeding (directly into the stomach through a tube in the skin) is now commonplace in community settings, whereas it used to be a hospital treatment. As a result, community nursing staff have much more complex caseloads. There is also greater complexity in the other forms of disability, as well as in the treatments that people are receiving and other technologies that are supporting them.

The Review Team was very struck by the experience of one on-call pharmacist whom they talked to. He was responsible for preparing the discharge medication for patients leaving hospital on a particular Bank Holiday weekend. He reported filling a doctor's prescription for 20 different medications for each of four patients. This strongly illustrates several points. Firstly, it is not right that such an excessive amount of medication should be routinely prescribed. It should be rigorously reviewed and adjusted. Secondly, it again shows the complexity and multiple conditions affecting many patients, who move regularly between hospital and community. Thirdly, it highlights the opportunity for a much stronger role for under-appreciated disciplines like pharmacy on the boundary between hospital and population.

The integration of health and social care means that the Review Team's discussions within Trusts necessarily took account of the important role of social care staff, and particularly social workers. They are a vital part of the workforce and although under equal pressure to their 
healthcare counterparts, the Review was encouraged to hear about the strong emphasis on professional development in Northern Ireland and the particular expertise in specialist areas such as adult safeguarding.

The knock-on effects of pressures in the hospital system for community services are not restricted to post-discharge matters. Many hospital departments are so pre-occupied with urgent work and the high volume of patients that they do not have time to provide proper responses when patients or their doctors make contact to ask about progress with an outpatient appointment or test results.

\subsubsection{High-pressure environments fuel risk to patients and sap morale}

The demand from patients who need emergency care, as well as those who require planned investigations and treatments, is extremely high. The pressures on emergency departments and hospital wards are very great. Over-crowded emergency departments and overflowing hospital wards are high-risk environments in which patients are more likely to suffer harm. This is because delays in assessment and treatment occur but also because staff have to make too many important and difficult decisions in a short space of time - what psychologists call cognitive overload. That they will make mistakes and misjudgments is inevitable, and some of them will be in life-and-death areas. Experience in other safety-critical industries, and research, shows that high-pressure, complex, and fast-moving environments are dangerous. If inadequate staff levels are added to the mix, risks escalate further.

The Review met with many groups of health and social care staff, speaking on condition of anonymity. They are overwhelmingly conscientious people who feel deeply for their patients and want to excel in the care that they deliver. Yet, the workloads in some situations are unacceptably high; so too are stress levels.
The stress comes not only from the large numbers of cases per se, but much more from the feeling of staff that they are not giving patients the quality of care they were trained to deliver. There is guilt too in knowing that they are forced to compromise their standards to levels that they would not accept for their own families. The phrase "doing just enough" was repeatedly used in the Review's meetings with front-line staff. There are extra pressures for some groups of staff. Doctors in training can find themselves in situations that are beyond their competence and experience. Sometimes they can call on back-up from senior staff, sometimes they have to do their best until the morning or Monday comes. Some nurses can find themselves dealing with an unacceptably large number of patients on a hospital ward at night. They too feel that they are having to lower their professional standards. This assessment is not based on isolated anecdotes but much more widespread and consistent accounts.

\subsubsection{Transformation efforts are moving slowly}

Transforming Your Care began as a substantial review of health and social care provision in Northern Ireland, commissioned in 2011. The review was led by the then-Chief Executive of the Health and Social Care Board, supported by an independent panel. It was a strong, forwardthinking piece of work.

The whole of the United Kingdom, like most developed countries, has a fundamental problem: the health and social care system that it has is not the health and social care system that it needs. The pattern of ill-health in the population has changed substantially since the systems were founded, and the systems have not changed to keep up. The Transforming Your Care review set out a convincing case for change. It described inequalities in health, rising demands, and a workforce under pressure. It particularly established that Northern Ireland has too many acute hospitals 
- that elsewhere in the United Kingdom, a population of 1.8 million people would likely be served by four acute hospitals - not the 10 that Northern Ireland had.

Transforming Your Care set out a broad new model of care, which aimed to be tailored to today's needs and person-centered. In practical terms, its most substantial proposal was to move £83 million away from hospitals and give it to primary, community and social care services.

Those interviewed by this Review Team unanimously supported the need for this initiative. The widespread feeling, though, is that Transforming Your Care is simply not being implemented.

As a result of weak communication and little action, there is substantial skepticism about Transforming Your Care. The Review Team heard it variously referred to as "Transferring Your Care", "Postponing Your Care", and even "Taking Your Chances". One of its central concepts, 'shift left', is viewed particularly warily. Carers see it as a euphemism for dumping work onto them; general practitioners likewise. Those working in the community see their workload increasing, and worry that there is no clarity at all about what the overall care model is supposed to be.

The frustrations of the general practitioner community in Northern Ireland that Transforming Your Care has not worked, is not properly planned nor funded, has led them to take matters into their own hands and form federations. General practices themselves are financially contributing to these, in a move to establish community-centered care pathways.

The needs that Transforming Your Care sets out to address are becoming ever more pressing. Its implementation needs a major boost in scale and speed, and communication needs particular attention.

\subsubsection{An under-powered system of commissioning}

At 1.8 million, the population of Northern Ireland is relatively small to justify what is a quite intricately designed health and social care management structure. In addition to the Department of Health, Social Services and Public Safety, there are six Trusts, a Health and Social Care Board with five Local Commissioning Groups, a Public Health Agency, and several other statutory bodies.

A central feature is the split between care providers and commissioners, which increases the complexity of the system and its overhead costs. This began life as the socalled purchaser-provider split, introduced by Margaret Thatcher's government in the late1980s. In various iterations, it has remained a feature of the NHS ever since. The introduction of a purchaser-provider split was originally intended to create a competitive "internal market' to drive up quality and so increase value for money. However, the scope for genuine competition has always been very limited. The term 'commissioning' subsequently superseded 'purchasing'. Commissioning involves a wider set of functions - assessing need and planning services accordingly, and the use of financial incentives to intentionally drive the system's development relating to the type of services provided, their quality and their efficiency.

Within the United Kingdom, the English NHS has the most developed commissioning system. NHS England, the national commissioning board, is now separate from the central government Department of Health. It is a pure commissioning organisation, completely free from overseeing the performance of Trusts. Its only relationship with the provider side of the market is through the commissioning process. It devolves the vast majority of funds to local Clinical Commissioning Groups (of general practitioners) that make decisions about the allocation of money against a national 
framework of policies and goals. Services are priced under a tariff system. This tariff has become increasingly complex, to facilitate locally agreed variation and to incorporate payfor-performance elements.

There are several contextual differences between England and Northern Ireland, of which the most obvious is population size. In England, the overhead costs associated with establishing and administering a complex tariff system are essentially divided between 53 million people. With a population one-thirtieth the size, the cost per head of running a similar system in Northern Ireland would be difficult to justify.

The problem for Northern Ireland is that it has gone just partially down the commissioning path. It does not have the benefits of a sophisticated commissioning system, yet has the downside of increased complexity and overhead costs. The worst of both worlds.

Northern Ireland has no service tariffs. The Health and Social Care Board allocates money by a process akin to block contracting. This approach was abolished years ago in England because it was considered old-fashioned, crude and not conducive to achieving value for money. Fully developed tariff systems reimburse providers on a case-by-case basis, with the amount paid dependent on the diagnosis or the procedure undertaken, the complexity of the patient and, in some cases, measures of the quality of care. In Northern Ireland, the funding system is far more basic. Staff the Review Team spoke to believed that it makes no distinction, for example, between a cystoscopy la simple diagnostic procedure, usually a day case) and a cystectomy (a complex operation), a clear absurdity if true.

Northern Ireland's five Local Commissioning Groups are not like England's Clinical

Commissioning Groups. The Local

Commissioning Groups have a primary focus on identifying opportunities for local service improvement. They have very few resources and, in effect, are advisers and project managers rather than commissioners. England's Clinical Commissioning Groups, by stark contrast, have a high degree of control over resource allocation.

It is imperative, somewhere in the system, for needs to be assessed, services planned and funds allocated. Whichever part of the system is responsible for this must be sufficiently resourced to do it well - arguably, the Health and Social Care Board is currently not.

The Northern Ireland system would benefit from stronger thought- leadership from within. There is no established health and social care think-tank, and some key disciplines such as health economics are not strongly represented.

Northern Ireland could choose to go down any number of different routes. It could strengthen the current Health and Social Care Board, particularly to create a tariff that includes a strong quality component. Alternatively, it could devolve budgetary responsibility to the five Trusts, making them something akin to Accountable Care Organisations in other countries, responsible for meeting the health and social care needs of their local population. The Trusts would then buy in primary care services, and contract between themselves for tertiary care services.

Recommending a commissioning model is beyond the scope of this Review. It is clear, though, that the Northern Ireland approach to commissioning is not currently working well, and that this is surely affecting the quality of services that are being provided. For that reason, the Review Team must recommend that this issue be addressed. 


\subsubsection{Who runs the health and social care system in Northern Ireland?}

It was instructive for the Review Team to have asked this question of many people. The question elicited a variety of answers, the common feature of which was that no one named a single individual or organisation. Indeed, most reflected their uncertainty with an initial general comment. Typical was a remark like: "The Minister has a high profile."

When pressed to directly answer the question: who runs the service? Their answers included: "The Minister", " The Permanent Secretary in the Department of Health", "The Chief Executive of the Health and Social Care Board", and "The Director of Commissioning of the Health and Social Care Board."

These responses reflect the complexity of the governance arrangements at the top of the health and social care system in Northern Ireland. They show that ambiguity has been created in the minds of people - both clinicians and managers - throughout the system.

The question of who is in charge is both simple and subtle. Whilst overall accountability versus calling the shots versus making things happen are aspects of governance that would have a single leadership locus in many places, this is not the case in Northern Ireland. There is no single person or place in the organisational structure where these things come together in a way that everyone working in the service, the public and the media clearly understand.

The present arrangements have evolved over time but the Review of Public Administration in 2007 led to many of them. Prior to this the Department of Health, Social Services and Public Safety was larger and oversaw four Commissioning Boards and 18 Trusts. There were highly-centralised control mechanisms and the service was subjected to many and frequent circulars and directives. Since then there has been a smaller Department of Health,
Social Services and Public Safety that is more focused on providing policy support to the Minister. A single Health and Social Care Board has been created from the previous four. The number of Trusts has been reduced from 18 to six, five organised to provide health and social care services by geographical area and the sixth an ambulance Trust for the whole region. Another important change has been the advent of a fully-devolved administration and the end of direct rule where power was in the hands of civil servants rather than elected local politicians. The lack of clarity about who is in charge is a major problem for Northern's Ireland care system. The difficulty is not that there is no figurehead, but that strategic leadership does not have the visibility of other systems. Without a clear leader, progress is piecemeal and change is hesitant and not driven through at scale - the Review Team was told "there are more pilots than in the RAF".

\subsubsection{Clarifying the role of healthcare regulation}

Aside from being commissioned by the Department of Health, Social Services and Public Safety to conduct occasional servicespecific inspections, the Regulation and Quality Improvement Authority has until now conducted a program of thematic reviews driving more at quality improvement than at regulation.

From 2015, the Minister has decided that the regulator should undertake a rolling programme of unannounced inspections of the quality of services in all acute hospitals in Northern Ireland. The Regulation and Quality Improvement Authority is being directed in this task to examine selected quality indicators in relation to triage, assessment, care, monitoring and discharge. As a result of this change, the regulator will reduce its normal annual programme of thematic reviews. 
These changes give the Regulation and Quality Improvement Authority a much stronger locus in the healthcare side of provision. However, this body has no real tradition of doing this kind of work, unlike its counterparts elsewhere in the United Kingdom. For example, in England, the various health regulators have evolved over a 15-year period with frameworks, methodologies, metrics and inspection regimes. For this reason, the Review is recommending that healthcare regulation in Northern Ireland is re-examined in the round, rather than approaching it piecemeal on an initiative basis. 


\subsection{INSUFFICIENT FOCUS ON THE KEY INGREDIENTS OF QUALITY AND SAFETY IMPROVEMENT}

The recognition that quality and safety should be a priority in the planning and delivery of health and social care arrived late to this sector in developed nations. Until the early 1970s, services operated on the tacit understanding that doctors' and nurses' education, training, professional values and standards of practice ensured that most care was good care. It was not until measurement of quality became more commonplace that it was realised that faith in this ethos had been badly misplaced. A series of scandals blew apart public confidence in the NHS. There were many victims, and it became clear that trust alone was not sufficient. Often, such events depicted cultures in some health and social care organisations in the United Kingdom and other countries that had tolerated poor practice and even sought to actively conceal it.

Organised programmes to assure quality and improve it initially came into healthcare through approaches developed in the industrial sector, notably total quality management and continuous quality improvement. Until 1998, there had never been a framework to progress quality and patient safety in the United Kingdom's NHS. From that time, a comprehensive approach was introduced with: standards set by the National Institute for Clinical Excellence and in National Service Frameworks; a programme of clinical governance to deliver assurance and improvements at local level backed up by a statutory duty of quality; and, inspection of standards and clinical governance arrangements carried out by the Commission for Health Improvement. These roles have changed over time. Some still cover all, or most, of the United Kingdom, whilst others have been taken up differently in the four countries.
Much recent commentary on the NHS in the United Kingdom has focused on whether its leadership is really serious about quality and safety. There is a widespread view within the service that financial performance and productivity are what really matter to managers, despite what might be in the mission statements of their organisations. This came home to roost in the scandalous events at the Mid-Staffordshire NHS Trust in England where the Francis Inquiry heard that concerns about quality were downplayed against financial viability in the pressure to gain Foundation Trust status.

A key consideration in quality and safety of healthcare is whether it is embedded in the mainstream at all levels. Up until the late1990s, it was largely the domain of academics and enthusiasts. Since then, those who are fully committed to its underlying principles and goals have increased in number. However, it is still debatable what proportion of board members, management teams, and clinical leaders are 'card-carrying' quality and safety enthusiasts.

Prominent in international experience are four essential ingredients to improving the quality and safety of care. These are: clinical leadership, cultural change, data linked to goals, and standardisation. In Northern Ireland seeds of each can be found, but none is blossoming. This is substantially holding Northern Ireland's care system back from achieving its full potential.

\subsubsection{Clinical leadership}

A crucial test of the strength of the quality and safety system is the extent of clinical engagement. This is partly a question of hearts and minds but also a case of knowledge, skills and the philosophy of clinical practice.

The quality and safety of care will only get better if those who deliver the care are not only involved in improving it, but are leading the improvement effort. In the very best healthcare 
systems in the world, clinicians are in the driving seat, supported by skilled managers. Traditionally, doctors, nurses and other health professionals have seen their duty to the patient in front of them. Rightly, this remains the important primary requirement for establishing a culture of good clinical practice. However, this is not enough to enable consistently high standards of care, nor to make care better year-on-year. This requires a paradigm shift in clinical practice, a different mission of practice, so that all healthcare professionals see the essence of their work not just in the care of individual patients but in ensuring that the service for all their patients reaches a consistently high standard and that opportunities for improvement are identified and taken. Accomplishing this is not easy. Clinicians will point out that their workloads are too heavy to make time to reflect on these wider considerations or that they do not have access to reliable data to allow them to compare their service to best practice or that they have not had training in quality and safety improvement.

Clinicians need to step forward to lead. This involves expanding their sense of responsibility beyond the individual patient in front of them to the system as a whole. When clinicians do step forward, they need to be supported. They need to be given responsibility and resources. They need to be given training, because leading improvement is technically and emotionally difficult.

In Northern Ireland, the Review Team met a small number of talented clinicians who have decided to step forward, and who are succeeding in leading positive change. The Review Team met many more clinicians who have tried to engage with 'management' in the past, have been knocked back, and have given up trying. There are many great ideas lying latent in the heads and hearts of clinicians, untapped by the system. The Review Team saw some effort, particularly in the South Eastern Trust, to provide clinicians with the skills that they need to lead improvement projects. Across the system as a whole though, the scale and scope of these is nowhere near what is needed.

\subsubsection{Cultural change}

Culture determines how individuals and teams behave day to day. It determines how clinicians view and interact with patients; whether they consider harm to be "one of those things", "the cost of doing business", or a feature of healthcare that, with effort, can be banished; whether they react to seeing problems in the system by complaining, or by taking on responsibility for fixing them.

All healthcare systems in the world realise the importance of culture. The difference between the best and the rest is what they do about this. The very best do not hope that culture will change; they put major effort into actively changing it. Their approach is not light-touch or scattergun; they see changing culture as a central management aim.

The Cleveland Clinic in the United States of America, for example, set out to improve patient experience, most of which is determined by how staff behave towards patients. The Clinic's management wanted all staff to better work as a team, and to see their role as being important for patient care - from doctors and nurses, to cleaners, receptionists and electricians. They designated them all 'caregivers'. All 40,000 caregivers attended a series of half-day training sessions, designed to build their practical communication skills and their awareness of self, others and team. They made patient experience scores widely available - ranked by doctor, by hospital, and by department. These efforts have continued for several years. In 2013, the Chief Executive's annual address to all caregivers included a powerful video about empathy. It has since been viewed 1.8 million times on YouTube. In short, the Cleveland Clinic made a major concerted effort to make patient experience important to all who work there. 
It has paid off. With staff now more engaged than ever, the Cleveland Clinic has been able to move on to making safety and other elements of quality a crucial part of the culture too.

In Northern Ireland, as in many places, no effort has been made to influence culture on anything like this scale. Many people in the system are able to describe the culture, and many cite it as important. Scattergun efforts are made - a speech here, an awards ceremony there - but shifting culture is hard, and scattergun will not do it. Culture is viewed with a degree of helplessness - but the evidence from elsewhere is that it can be changed, and that doing so is powerful.

\subsubsection{Data linked to goals}

The importance of data and goals are news to nobody. Yet in Northern Ireland, as in too many other healthcare systems, data systems are weak and proper goals are sorely lacking.

Improving healthcare requires clear and ambitious goals. It requires a statement that preventable harm will be reduced to zero, or that the occurrence of healthcare associated infections will be cut in half within a year. Management guru Jim Collins would call these BHAGs - Big Hairy Audacious Goals. They are goals that are at once exciting and scary. They get people interested and motivated. They are the kind of goals that Northern Ireland should be setting for its care system.

If the goal is the destination, strong data are the sat nav. They show the current position in a form that provides useful information for action. Too often, data show where the system was over the last three months, or what performance has been across large units. They need instead to show the situation in real-time, or as near to it as possible. And they need to show performance at the very local level.
As with culture and leadership, data capability is an area that the best care systems in the world have invested in heavily. They have online dashboards that enable all aspects of the system to be measured, understood, and therefore managed. In comparison, Northern Ireland (and many other places) has a care system that is being managed as if through a blindfold. Investment in information technology is crucial and, if done intelligently, will pay dividends.

\subsubsection{Standardisation}

Doctors generally dislike standardisation (nurses warm to it more), but it is a crucial part of improving the quality and safety of healthcare.

One healthcare standardisation tool is the World Health Organization's Safe Surgery Checklist. Modelled after the checklists that pilots use throughout every flight, it lists a series of simple actions that should be taken before the patient receives anaesthetic, before the operation starts, and before the patient is moved from the operating theatre. Each item on the list is something blatantly obvious - checking the patient's identity, confirming the type of operation that is planned, and so forth. Without the checklist, each of these things is done most of the time - but not all of the time. The checklist ensures that they are done all of the time - to avoid the occasional instance, as happens, in which nobody properly checks the operation type, and the patient has the wrong operation.

Care bundles are a concept that in recent years have brought higher quality to the areas of care where they have been used well. They help clinicians to reliably give every element of best practice treatment for common conditions such as pneumonia. The evidence is clear: they save lives. Without them, patients get best, safest practice only some of the time and those who do not are the unlucky ones who can suffer greatly as a consequence. 
Checklists and care bundles are not widespread in healthcare primarily, because they are counter-cultural. Doctors' training, in particular, emphasises the importance of retaining knowledge, of autonomy, and of variation between patients. All of these go against the idea of standardisation.

The concept of standardisation does not just relate to novel methods like checklists or care bundles. It is also concerned with all patients with a particular disease receiving a consistent process of care based on best practice internationally. The idea that people with conditions like bowel or oesophageal cancer should be receiving different treatment based on clinical preference or where they live is a disgrace. Healthcare should not be a lottery.

The best healthcare systems in the world have a high degree of standardisation. Not for everything - but for the areas of care where the evidence shows that it makes a difference. They have a substantial number of care pathways, checklists, and care bundles. This does not leave the clinicians without a job - far from it. Their judgement is vital in deciding which pathway, checklist or care bundle to use, and in spotting the cases in which a standard approach is not appropriate. They still spend the majority of their time working without reference to any of these things, but use them whenever they are needed.

Northern Ireland has some good examples of work in this area, including the rollout of a National Early Warning System for acutely ill patients, a care bundle for sepsis, an insulin passport, and regional chest drain insertion training. However, the opportunity for standardisation is much greater and needs to be applied at a more fundamental level, which influences the model of practice beyond this series of individual initiatives. There is not yet a critical mass of clinicians clamouring for more standardisation. There are multiple examples of different Trusts approaching the same clinical scenario in different ways, and wanting to retain their autonomy to do so. If Northern Ireland wants to be anything like as good on safety, clinical effectiveness and patient experience as the Cleveland Clinic and other centres of excellence, it needs to be more open to big change.

\subsubsection{The recipe for success}

There is little doubt that quality and safety are not fully embedded in the planning, design and delivery of services in Northern Ireland. More sleep is lost over budgets than about whether patients are treated with dignity and respect, whether outcomes of care are genuinely world class and whether patients are properly protected from harm when they are being cared for.

Four vital, and often superficially treated, ingredients for quality and safety improvement are: clinical leadership, cultural change, data linked to goals, and standardisation. They are highly inter-linked.

The Northern Ireland care system is not seeing the wood for the trees on these ingredients. The Quality 2020 strategy cites them land does set some big goals), but they are not held as central and are therefore somewhat lost. They need to be given far more prominence, because they form the bedrock on which all quality and safety improvement is built.

With focused effort, Northern Ireland could: build a cadre of skilled clinical leaders; develop a culture in which quality improvement is second nature; set big goals; establish the information technology systems required to measure quality locally and in real-time; and standardise processes substantially. If the care system makes these activities central to its quality and safety efforts, improvement will follow and will flourish. Without building this bedrock, no other efforts to improve quality and safety will gain any significant purchase. 


\subsection{EXTRACTING FULL VALUE FROM INCIDENTS AND COMPLAINTS}

Most patient safety programmes have at their core a process to capture and analyse errors and accidents that arise during the provision of care. This is based on the longestablished premise that only by learning from things that go wrong can similar events be prevented in the future. To some extent, this draws on the experience of other industries that have successfully reduced accidents and risk year-on-year. This thinking has led to the establishment of incident reporting systems in health services across the world, some operating only at the level of healthcare organisations, some encompassing whole countries and some restricting reports to those within one field of medicine (e.g. surgery).

It is not always appreciated that reporting of incidents (which can be voluntary or mandatory) is only one way of assessing harm in the care of patients. Numerous other approaches have been used, including: prospective observation of care processes; trigger tools involving retrospective case note review; expert case note review; Hospital Standardised Mortality Ratios (and similar metrics); and mining electronic hospital databases.

Alongside Northern Ireland's incident reporting systems runs a complaints system. Globally, surveys have consistently shown that what patients want from a complaints system are: an explanation, an apology, and a reassurance that improvements to the service will be made based on their experience. Other jurisdictions have found that the features of a good complaints system are: satisfactory local resolution of the majority of complaints; speedy response times; excellent communication with patients; good record keeping; apologies made in-person by the senior staff involved not on their behalf; accurate monitoring of the numbers and categories of complaint; effective learning at the local and systemic level.
All these systems have a common primary purpose: to improve the quality of care, and to reduce avoidable harm.

\subsubsection{Incident reporting elsewhere}

Globally, incident reporting systems vary greatly in: the nature of the data captured, the extent of public release of information, whether reporting is voluntary or mandatory, and the depth of investigation undertaken.

Most reporting systems start by defining in general terms what should be reported. Terminology varies; adverse event, incident, error, untoward incident are all in common use internationally. The epithet serious can be applied to any of the terms. The largest national system in the world was established in the NHS in England and Wales as a result of the report An Organisation with a Memory. From 2004 until recently, it was run by an independent body, the National Patient Safety Agency, and is called the National Reporting and Learning System. NHS staff are encouraged to make an incident report of any situation in which they believe that a patient's safety was compromised.

In this system, a "patient safety incident" is defined as "any unintended or unexpected incident which could have, or did, lead to harm for one or more patients receiving NHS care." Reports are first made to a local NHS organisation and then sent in batch returns by the local risk manager to the national level. Staff make a small number of reports electronically directly to the National Reporting and Learning System. The information required covers: demographic and administrative data; the circumstances of occurrence; a categorisation of causation; an assessment of the degree of harm as "no", "low", "moderate", "severe", or "death"; and action taken or planned to investigate or prevent a recurrence. These data are captured in a structured reporting form, but there is also a section of free text where the reporter is asked to describe 
what happened and why they think it happened. Data are anonymised to remove the names of patients and staff members.

In just over a decade, covering the NHS in England and Wales, nearly 10 million patient safety incidents have accumulated in this database. Since 2012, it has been mandatory to report all cases of severe harm or death. It remains voluntary to report all other levels of harm.

During the period of its existence, the National Patient Safety Agency in England and Wales issued 77 alerts and many other notices about specific risks, most of which had been identified by analysis of patient safety incident reports. New arrangements for issuing alerts are in place following the abolition of the National Patient Safety Agency.

This system of incident reporting in England and Wales holds a huge amount of data but only a small proportion of it is effectively used. It is currently being reviewed and is unlikely to continue in exactly the same way.

Worldwide, the problems associated with incident reporting are remarkably consistent, whatever system design is adopted. Firstly, under-reporting is the norm, although its degree varies. This seems to depend on the prevailing culture and whether incidents are seen as an opportunity to learn or as a basis for enforcing individual accountability and apportioning blame. It also depends on staff perceptions about the difference their report will make and how easy it is for them to convey the information that they are required to. Reporting rates are much lower in primary care services than in hospitals. Secondly, given the volume of reports made, there is often insufficient time, resource and expertise to carry out the depth of analysis required to fully understand why the incident happened. Thirdly, the balance of activity within reporting systems goes on collecting, storing, and analysing data at the expense of using it for successful learning. Indeed, there are relatively few examples worldwide of major and sustained reductions in error and harm resulting because of lessons learnt from reporting.

\subsubsection{Incident reporting in Northern Ireland}

Incident reporting began in the Northern Ireland health and social care system in 2004. Two categories of incident were established: an adverse incident and a serious adverse incident. The former were reported and investigated locally within each Trust. The latter were documented and investigated locally but also had to be reported to the Department of Health, Social Services and Public Safety. Staff make 80,000 to 90,000 adverse incident reports each year. Over 400 Serious Adverse Incident reports were made in 2013. In the five-year period from 2009, the number of Serious Adverse Incidents related to Emergency Departments rose from 8 to 36.

An adverse incident is defined as:

"Any event or circumstances that could have or did lead to harm, loss or damage to people, property, environment or reputation."

In 2010, major new guidance was issued passing responsibility for managing and further developing the serious adverse incident system to the Health and Social Care Board, where it remains to this day. Further guidance was issued in 2013 with new reporting rules.

To be regarded as a Serious Adverse Incident for reporting purposes, the incident must fall into one of the following categories: the serious injury or unexpected/unexplained death of a service user, staff member or visitor; the death of a child in health or social care; an unexpected serious risk to a service user and/or staff member and/or member of the public; an unexpected or significant threat to service delivery or business continuity; serious 
self-harm or assault by a service user, staff member, or member of the public within a healthcare facility; serious self-harm or serious assault by any person in the community who has a mental illness or disorder and is in receipt of mental health and/or learning disability services, or has been within the last twelve months; and, any serious incident of public interest.

Any staff member may report an adverse incident. The reporter is not asked to make a judgment about whether the incident meets the serious adverse incident criteria. A responsible manager makes it based on their reading of the incident and application of the guidelines. Any Serious Adverse Incident must be reported to the Health and Social Care Board within 72 hours. A subset of Serious Adverse Incidents must be simultaneously reported to the Health and Social Care Board and the Regulation and Quality Improvement Authority.

Trusts in Northern Ireland differ slightly in the procedure adopted for encouraging, receiving and investigating incident reports. Generally, all staff are encouraged to make reports as a way of making care safer. They complete an incident report and submit it to the Trust's risk management department so that it can be entered into the risk management database. Increasingly, more reports are being made online which cuts out the laborious form-filling which is an undoubted barrier to staff making a report and often leads to paper mountains in the risk management department. Trusts vary in the proportion of incidents that they investigate, the depth of that investigation and the extent to which action is agreed and implemented. Clinical governance committees (or their equivalents), sub-committees of the Trust board or the Board itself usually look at a selection of individual incident reports, at aggregated incident data or at both.
The number of Serious Adverse Incidents varies between Trusts (Figure 1). To some extent this reflects their differing number of patients. However, there is no way of knowing at present whether a higher level of incidents means that the organisation is less safe than others or that it is more safe and that its staff are more conscientious in making reports so that learning can improve patient safety. Whilst data are available on Serious Adverse Incident types, the categories and classifications used do not make it easy to aggregate data in a way that enables systemic weaknesses to be identified. Opportunities are therefore being lost for surveillance of patient safety across Northern Ireland.

The vast majority of Serious Adverse Incidents are reported by the five acute Trusts. Much smaller numbers are reported by the ambulance service and by primary care (Figure 2). The number of incidents reported has increased quite substantially from 2013 to 2014 (Figure 3). In part this is because of improved awareness of the reporting system. In part it is because the reporting criteria were changed most notably, requiring that all child deaths be reported. 
Figure 1. Serious Adverse Incident reports: by Trust

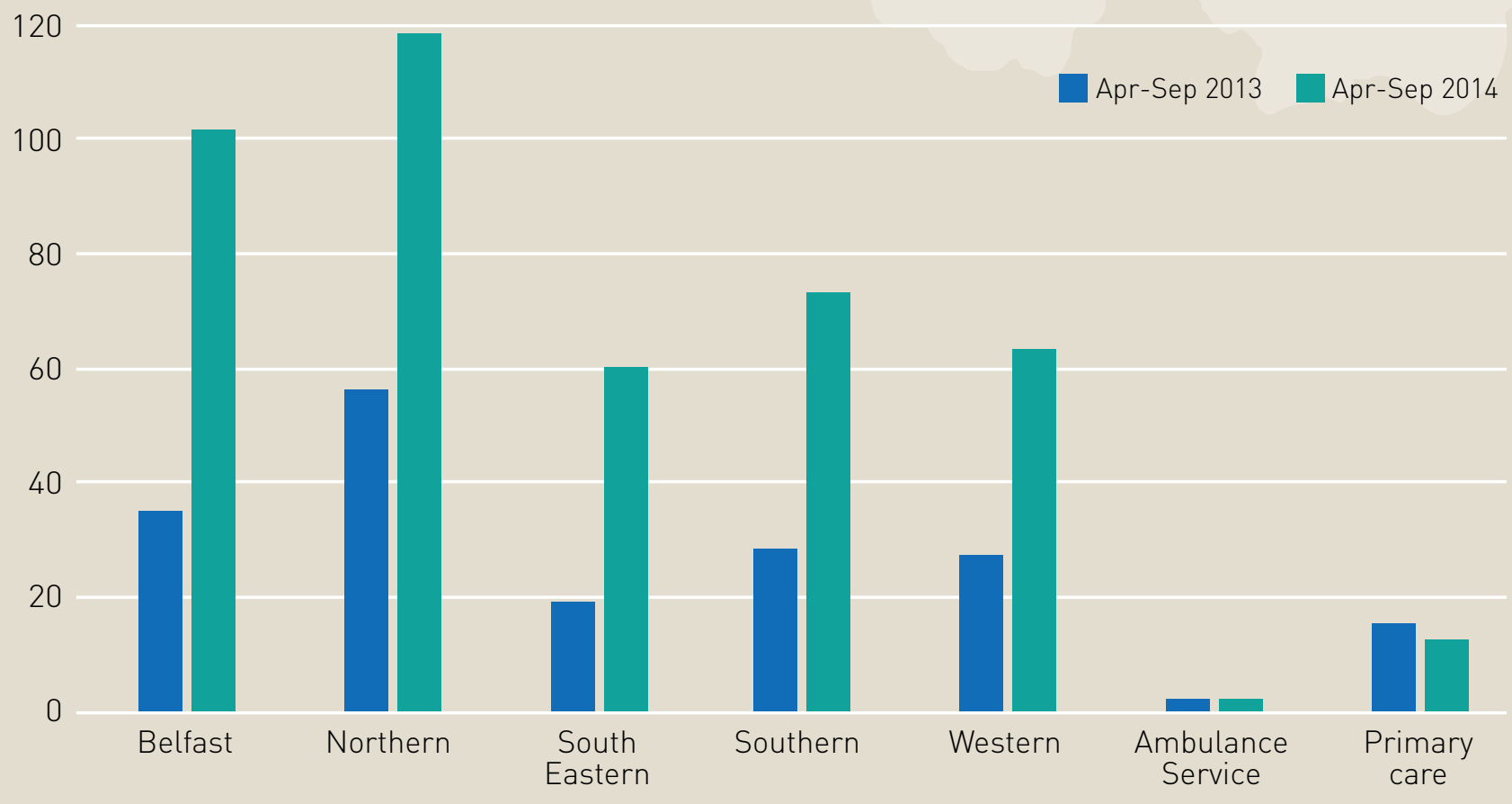

Health and Social Care Trusts

Figure 2. The great majority of Serious Adverse Incident reports are made by the Health \& Social Care Trusts

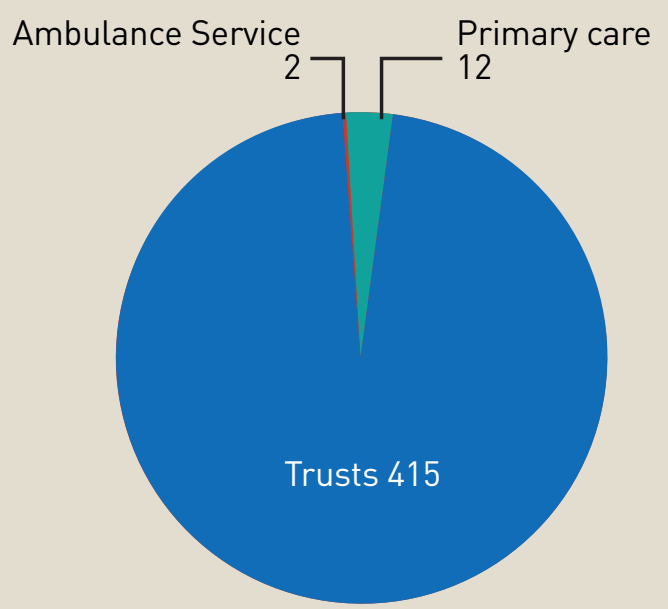

Serious Adverse Incident Reports, Apr-Sept 2014
Figure 3. Serious Adverse Incident reporting increased between 2013 and 2014.

Some of the increase was because reporting criteria changed, particulary introducing a requirement to report all child deaths.

500

Child deaths (New reporting requirements)

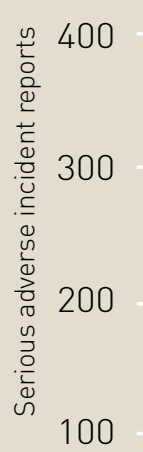

0

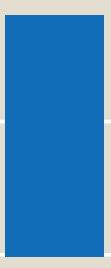

2013

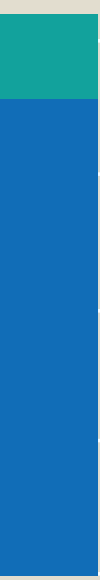

2014

April-September 
All Serious Adverse Incidents are investigated. The type (and therefore intensity) of the investigation should depend on the severity of the incident, its complexity, and the potential to learn from it. Three levels of investigation are stipulated:

- Level 1 involves a Significant Event Audit a method of assessing what has happened and why, agreeing follow-up actions, and identifying learning.

- Level 2 involves a Root Cause Analysis - a more detailed exercise to determine causation and learning, undertaken by a formal investigation team chaired by somebody not involved in the incident.

- Level 3 involves a full-blown independent investigation.

Most Serious Adverse Incidents start at Level 1 investigation, and may proceed to Level 2 or 3 if the Level 1 investigation suggests that this is necessary or would be useful. A minority start at Level 2 or 3 immediately, bypassing Level 1.

A Designated Review Officer, assigned by the Health and Social Care Board and Public Health Agency, provides independent assurance that an appropriate level of investigation has been chosen, and that it is conducted appropriately.

The process of dealing with Serious Adverse Incidents at the operational level of the service is very involved and highly regulated with little room for flexibility. There are a number of decision-making points at which important judgments must be made by staff on matters such as what level the incident falls into and whether to refer an incident to the coroner.

\subsubsection{Frustrations with the incident reporting system}

The staff who use the incident reporting system have concerns and frustrations. Firstly, at the policy level, the requirements to report Serious
Adverse Incidents places a considerable burden on them to complete forms and meet deadlines, with very little flexibility to deviate from the proscribed procedure. There is an acceptance by staff that it is important to document and investigate Serious Adverse Incidents but the pressure to complete all the steps of the process often means that there is no time to reflect on what can be learned so as to reduce risk for future patients. One of the Serious Adverse Incidents that the Review Team discussed with Trust staff had involved interviews with 34 different people. It was by no means the most complex incident that the Review Team heard about.

There is an almost universal view that the requirement to report and investigate all child deaths in hospital as Serious Adverse Incidents has been a retrograde and damaging policy decision. The consequence of it has been that, if a child dies from a cause such as terminal cancer or a congenital abnormality, a grieving family must be advised that there is to be an investigation. Inevitably, this strongly implies that the service has been at fault. Such an approach is not kind to such families, puts staff in a very difficult position, and diverts attention from the investigation of genuinely avoidable incidents involving the care of children. In a separate aspect of incident policy, many staff working within the mental health field have concerns about the inflexibility of the Serious Adverse Incident scheme as it applies to suicide of their patients. Whilst the time-scales for investigation impose a necessary discipline on the process generally, the range of factors, individuals and agencies that need to be part of the determination of the root causes of the suicide of a mental health patient are very great indeed. The pressure to adhere to statutory deadlines can mean that the work in such cases can sometimes be incomplete and so has limited value in preventing recurrences. 
Secondly, at the cultural level, some medical, nursing and social care staff are concerned that, in reporting an adverse incident, they will expose themselves to blame and possible disciplinary action. Junior doctors told the Review Team that making too many reports draws suspicion that they are trouble-makers and that an active interest in patient safety could damage their career prospects. They prefer to make their views on patient safety known through the medical trainee annual survey (Figure 4), where they can remain anonymous.

Figure 4. Percentage of medical trainees reporting concerns about patient safety and the clinical environment

\begin{tabular}{l|c|c|c|c|c}
\hline Trust: & Belfast & Northern & South Eastern & Southern & Western \\
\hline Patient safety & $6.5 \%$ & $6.8 \%$ & $3.0 \%$ & $4.7 \%$ & $3.2 \%$ \\
\hline Clinical environment & $2.8 \%$ & $3.6 \%$ & $0.8 \%$ & $1.4 \%$ & $0.4 \%$ \\
\hline Total & $9.3 \%$ & $10.4 \%$ & $3.8 \%$ & $6.0 \%$ & $3.7 \%$ \\
\hline
\end{tabular}

Source: General Medical Council National Training Survey 2013. Numbers are rounded.

These cultural barriers to reporting and learning are not unique to Northern Ireland. Creating a culture where the normative behavior is learning, not judgment, is very much the responsibility of political leaders, policy-makers, managers and senior clinicians. This does not mean that no-one is ever accountable when something goes wrong but it does mean that a proper regard should be given to the overwhelming evidence that a climate of fear and retribution will cause deaths not prevent them.

Thirdly, at the operational level, staff frustrations with the incident reporting processes range from the very practical, such as not being able to find the form necessary to make the report, to the deeper de-motivating features of the system such as never receiving any feedback or information on the outcome of the report that they had made. Other weaknesses of the process perceived by staff include: having little training in how to investigate properly, reporting an incident then being asked to investigate it yourself, and a tendency for investigations to descend into silos even though there might have been a multispecialty element to the patient's care.

\subsubsection{The complaints system in Northern Ireland}

Patients, their carers, and their families can make a complaint about the services received in person, by telephone or in writing. If the complaint concerns the health or social care services delivered by one of the six Trusts in Northern Ireland, a senior officer within the organisation will work with the staff involved in the person's care to investigate and produce a response. A letter from the chief executive officer of the Trust must go to the complainant within 20 working days. However, performance is suboptimal and very variable in this respect (figure 5). 
Figure 5. All Trusts are failing to meet the standard 20-day substantive response time for complaints (\% meeting standard shown; 2013-14)

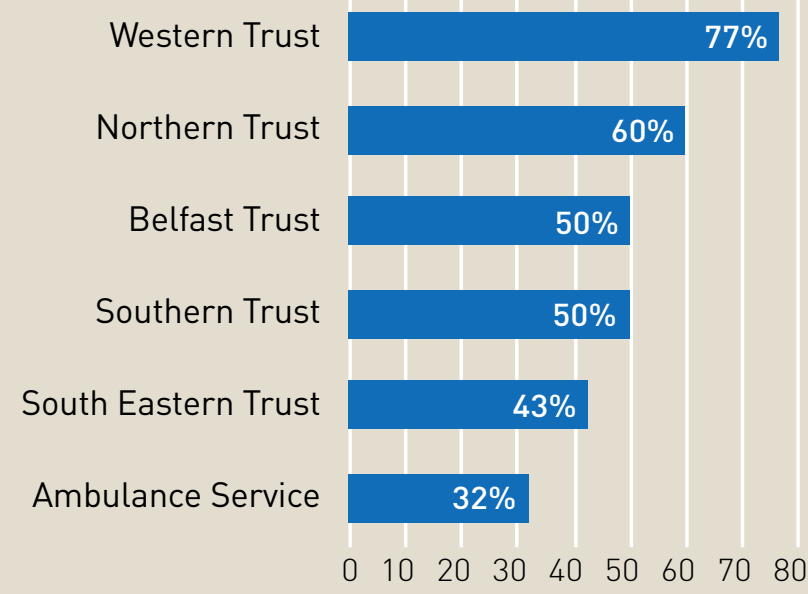

The best outcome is for the complaint to be resolved locally to the complainant's complete satisfaction. This is not always possible and if the complainant is not satisfied with the response, the complaint can be re-opened and further investigation can be undertaken or external advice sought. If this still does not resolve the complaint, the complainant can make a submission to the Ombudsman. He will look at whether the process of responding to the complaint was undertaken appropriately. He can also investigate the substance of the complaint but under present legislation, he cannot make these reports public. This bizarre situation means that the public is unaware of where standards have fallen short and what the Ombudsman thinks should be done.

An increasing number of people who have complaints contact The Patient and Client Council asking for help. The Council does not have powers to investigate complaints, only to provide support. Nearly 2000 complainants contacted the Council last year. Many such contacts were from people who had tried to navigate the complaints system alone and had had difficulties. The Patient and Client Council's involvement often helps in facilitating resolution of the complaint, sometimes by arranging meetings of the two sides.

Complaints about primary care are handled somewhat differently. They are raised with the Health and Social Care Board directly. The number of complaints from primary care is lower than might be expected. This may reflect the reluctance of patients to complain about a service that they are totally reliant on.

\subsubsection{Involvement of the coroner}

Northern Ireland, like elsewhere, is still grappling with a difficult question: what is the appropriate role for the Coroner in the investigation of deaths that may have been caused, at least in part, by patient safety problems? This is not an easy question. It is difficult to create guidance that precisely defines which deaths should be investigated by the coroner and which should not. And Coroner's inquests have major pros and cons.

When somebody dies and their care may have been perceived as poor, some families call for a Coroner's inquest. The positive elements of this are that the Coroner is independent of the health and social care system, has clear legal powers, and is skilled in the investigation of deaths.

On the other hand, conducting an inquest into every Serious Adverse Incident that results in a death would be a resource-intensive undertaking. It also may not result in the most effective learning. Few could honestly say that the courtroom environment does not intimidate them. It is not the easiest place to build a constructive relationship between the clinicians involved in the care of the deceased and the deceased's family. It is not the most conducive environment to open, reflective learning.

In cases of negligence or gross breaches of standards of care, it is very clear that referral to the Coroner is the most appropriate course. 
At the other end of the spectrum, in a few cases there is a Serious Adverse Incident at some point during a patient's care and this patient subsequently dies, but the death is entirely unrelated to the incident and so an inquest is really not warranted. In between these two extremes lies a substantial grey area, in which the relative merits of a Coroner's inquest and an internal Serious Adverse Incident investigation are debatable. This is not only the case in Northern Ireland, but across the United Kingdom as a whole (except that Scotland does not have a Coroner).

This is a complex issue. Currently only a subset of the deaths that could be the subject of a Coroner's inquest actually become so. Some are not reported to the coroner's office (largely appropriately, it seems) and some are discussed with the coroner's office but not listed for inquest. In other words, the judgments of clinicians and coroners' officers alike have a substantial bearing on which cases proceed to inquest. The subset of cases that end up in front of a coroner's inquest are also determined as much by family's wishes as by the content of the cases.

To some this may sound shocking but, given the complexity of the issues involved, the status quo is not entirely unreasonable and is in line with practice internationally. But the status quo is certainly not ideal. There is substantial room for improvement, so that the coroner can more optimally contribute to the system's learning.

\subsubsection{Redress}

The creation of financial, and other new, forms of redress would have to be linked to the handling of complaints, incidents and medical negligence claims in a whole systems manner. This is a highly complex area that was extensively examined in England in the report Making Amends. In the end, the central idea of introducing some payments for victims of harm and recipients of poor quality care, as well as potential litigants, was not taken forward. There were sound principles behind the proposals, but there was a leap-in-thedark element too. Priority was given instead to action to improve the quality and safety of care and to improve responses to complaints. However, one of the other proposals of Making Amends, the introduction of a Duty of Candour, is finally being implemented in England. The Review Team considers that priority in Northern Ireland should be given to the areas covered by its recommendations, to making important changes to generate safer higher quality care, rather than embarking on new policies for redress, including financial compensation.

\subsubsection{The nature of learning}

The whole question of how learning takes place in healthcare through the scrutiny and analysis of incident reports or through their investigation has been little debated. Indeed, the term learning itself is very loosely applied in this context. Strictly applied, it would mean acquiring new knowledge from incidents about how harm happens. Yet, the way in which the word learning is repeatedly used in the context of patient safety is more than increasing understanding. It implies that behaviour will change or actions will be taken to prevent future harm. Unfortunately, although there are some exceptions, there is little evidence that major gains in the reduction of harm have been achieved in Northern Ireland or in many other jurisdictions through the so-called learning component of patient safety programmes.

In Northern Ireland, the main formallyidentified processes for reducing risk or improving patient safety, aside from action plans derived at Trust level, are:

- the production of learning letters

- the bi-annual Serious Adverse Incident Learning Report

- the circulation of newsletters such as Learning Matters

- thematic reviews

- training and learning events 
- implementing the recommendations of reviews and inquiries

- disseminating alerts and guidance imported from other parts of the United Kingdom or further afield.

On many, perhaps most, occasions when something goes wrong, the potential for learning from this is very rich indeed. This potential too often goes unrealised. This is a problem not just in Northern Ireland, but in care systems worldwide.

Three features determine the extent to which investigation of an adverse event results in risk being reduced:

- How deep the investigation gets, in understanding the true systemic issues that helped something go wrong

- How systemic the investigation's focus is, in considering where else a similar problem could have occurred beyond the local context in which it did occur

- How strong the corrective actions are in actually, and sustainably, reducing the risk of a repeat

The first of these, depth of investigation, is done reasonably well. A decade ago, harm was often put down to 'human error'. There is now far greater recognition that this is a superficial interpretation - that there are almost always problems within the system which not only allowed that harm to occur but made it more likely. The technique of root cause analysis is widely used in Northern Ireland, and helps to uncover some of the causal elements. Often, though, it does not find the deeper reasons. This is partly because of the time pressures to finish the investigation, partly because not all staff have had the necessary training to do this deeper analysis, and partly because of a lack of human factors expertise in the process. Also, many hospital incidents involve primary care in the chain of possible causation, yet primary care staff play a minor, or no, role in many investigations.
In relation to the systemic view, when a problem occurs, there is too great a tendency to investigate that specific problem, without looking for the broader systemic issues that it highlights. Problems are often addressed in the department where they occur, without asking whether they could have occurred in other departments, for example. Similarly, if a medication incident occurs, there is a tendency to fix the problem for that medication, without looking at whether there is a problem for similar medication or routes of administration.

This narrow, reactive approach fails to make full use of incident reports. In short, it reflects an erroneous assumption that the system as a whole is working fine, and that the problems that allowed the event to occur are specific, local ones. This is not the case. There are systemic problems through the health and social care system. Incidents of harm are distributed largely by chance - by location and by type. Fixing each specific problem is like playing "Whack-A-Mole" it does not get to the nub of the issues.

The ultimate aim of investigation is to reduce the risk of harm, not simply to understand what went wrong. Corrective action is too often inadequate. There is no automatic link between understanding what went wrong and being able to reduce the risk of it happening again. Indeed, making the leap between investigation and risk reduction is really very challenging.

In Northern Ireland, the action lists that are generated by Serious Adverse Incident investigation commonly feature plans of the following kinds:

- Making staff aware that the incident took place

- Explaining to staff what went wrong

- Circulating a written description of the incident and actions taken to other parts of the health and social care system to share the learning 
Such information sharing actions should form part of the plan but they do not amount to systemic measures that will reliably and significantly reduce the risk to patients.

Research and experience outside health care has shown that safety comes down to appreciating that big improvements are not made by telling people to take care but by understanding the conditions that provoke error.

Action plans often also feature some change to current paperwork or introduction of new documentation. This, too, is very reasonable but often has a weak impact on outcomes. It also has the important downside that mounting paperwork reduces the time for patient care and introduces complications of its own.

So what do strong corrective actions look like? Technological solutions have an important role to play. Electronic prescribing systems, patient monitoring systems, and shared care records can address multiple patient safety issues simultaneously lalthough their implementation and use is not without risk). Policies, rules, and checklists can also be useful, but are easy to implement badly and more difficult to implement well.

As discussed earlier in this Report, one area of high potential is the use of standardisation of procedure. It is underutilised in healthcare worldwide but where it is applied it has brought results. Standardisation of procedure is a mainstay of safety assurance and improvement in other sectors.

In large part, though, healthcare systems worldwide are not yet good at implementing solutions that will truly reduce risk. It is not the case that Northern Ireland is lagging behind but that Northern Ireland is struggling with this problem alongside other countries.
Identifying the systemic issues and identifying strong corrective actions: each of these is tough; an art and a science in itself; an area in need of intense and rigorous study. Until these issues are tackled head on, in Northern Ireland and elsewhere, the system's learning when things go wrong will fall short.

When something goes wrong, patients and families ask for reassurance that it will not happen again. As it stands, nobody can honestly provide this reassurance. In fact, it is difficult even to say that the risk has been significantly reduced - let alone to zero. This needs to change.

\subsubsection{Strengths and weaknesses of Northern Ireland's systems for incident reporting and learning}

No system of reporting and analysing patient safety incidents is perfect. In an ideal world, all events and occurrences in a health service that caused harm or had the potential to cause harm would be quickly recognised by alert, knowledgeable front-line staff who would carefully document and communicate their concern. They would be enthusiastic about their involvement in this activity because they would have seen many examples of how such reports improved the safety of care. The resulting investigation would be impartial and multi-disciplinary, involving expertise from relevant clinical specialties but, crucially, also from other non-health disciplines that successfully contribute to accident reduction in other fields of safety. Investigation would be carried out in an atmosphere of trust where blame and retribution were absent, and disciplinary action or criminal sanctions would only be taken in appropriate and rare circumstances. Action resulting from investigation would lead to redesign of processes of care, products, procedures and changes to the working practices and styles of individuals and teams. Such actions would usually lead to measurable and sustained reduction of risk for future patients. Some types of harm would be eliminated entirely. 
Very few, if any, health services in the world could come anywhere near to this ideal level of performance in capturing and learning from incidents of avoidable harm. This is so for all sorts of reasons ranging from an insufficiency of leaders skilled and passionate enough to engage their whole workforces on a quest to make care safer, through an inability to investigate properly the volume of reports generated, to the weak evidence-base on how to reduce harm.

The system of adverse incident reporting in Northern Ireland operates to highly-specified processes to which providers of health and social care must adhere. The main emphasis is on the
Serious Adverse Incidents. The requirements laid down for reporting, documenting and investigating such incidents together with the rules for communicating about them and formulating action plans to prevent recurrence have created an approach that has strengths and weaknesses (Figure 6). In general, the mandatory nature of reporting means that there is likely to be less under-reporting than in many other jurisdictions. However, staff in Trusts must exercise judgment on whether to classify occurrences of harm as Serious Adverse Incidents. Whether they always make the right decision has not been formally evaluated. The Review did not find any evidence of suppression or cover-up of cases of serious harm.

Figure 6. Serious Adverse Incident reporting system in Northern Ireland: Strengths and weaknesses

\begin{tabular}{l|l|l} 
Dimension & Strengths & Weaknesses \\
\hline Accountability & $\begin{array}{l}\text { Absolute requirement to report and } \\
\text { investigate }\end{array}$ & $\begin{array}{l}\text { Creates some fear and } \\
\text { defensiveness }\end{array}$ \\
\hline Coverage & $\begin{array}{l}\text { Relatively high for serious } \\
\text { outcomes }\end{array}$ & $\begin{array}{l}\text { Less attention given to incidents } \\
\text { with lower harm levels }\end{array}$ \\
\hline Timescales & $\begin{array}{l}\text { Clear deadlines for investigation } \\
\text { and communication }\end{array}$ & $\begin{array}{l}\text { Pressure to meet deadlines leaves } \\
\text { little time for reflection }\end{array}$ \\
\hline Staff engagement & $\begin{array}{l}\text { Reasonable depth with frequent } \\
\text { root cause analysis }\end{array}$ & $\begin{array}{l}\text { Quality variable and little use of } \\
\text { human factors expertise }\end{array}$ \\
\hline Patient and family involvement & $\begin{array}{l}\text { All appear to understand the } \\
\text { importance of reporting }\end{array}$ & $\begin{array}{l}\text { Do not often see the reports } \\
\text { translating into safer care }\end{array}$ \\
\hline Learning & $\begin{array}{l}\text { Requirement to communicate } \\
\text { reinforced by checklist }\end{array}$ & $\begin{array}{l}\text { Often creates tension and little } \\
\text { ongoing engagement }\end{array}$ \\
\hline & $\begin{array}{l}\text { Specified action plan required in } \\
\text { every case }\end{array}$ & $\begin{array}{l}\text { Not clear whether action is } \\
\text { effective in reducing future risk }\end{array}$ \\
\hline
\end{tabular}


Tight time-scales are laid down for the various stages of handling a Serious Adverse Incident. These generally add a necessary discipline to a process that in other places can become protracted or drift off-track. There is a need, though, for some flexibility where an investigation requires more time. This is particularly so in the mental health field where the avoidable factors in a death can be very complex and are only discernible after interviewing very many people.

It is important to recognise that, whilst almost all of the experience and research literature is about patient safety, Northern Ireland has an integrated health and social care system. Social care in the United Kingdom has its own traditions in recognising, investigating and learning from episodes of serious harm involving those who use its services; the fields of child protection and mental health exemplify this. It is not entirely straightforward to integrate incidents in social care into the overall patient safety approach but the essential principles and concepts are little different.

The Northern Ireland health service falls short of the ideal just as do most other parts of the United Kingdom and many other places in the world. In all of these places, including Northern Ireland, patients are dying and suffering injuries and disabilities from poorly designed and executed care on a scale that would be totally unacceptable in any other high-risk industry.

The Northern Ireland approach to incident reporting and learning does not make its services any less safe than most of the rest of the United Kingdom or many other parts of the world. However, this should not be a reason for comfort, nor a cause for satisfaction.
The current requirement for all child deaths to be reported and managed as serious adverse incidents seems to be doing far more harm than good. It is distressing for families, burdensome for staff, and is not producing useful learning.

The ethos of improving safety by learning from incident investigations needs to shift:

- Away from actions that only make a difference in the particular unit where the incident occurred, towards actions that also make a difference across the whole of Northern Ireland

- Away from actions that only target that particular incident, towards actions that also reduce the risk of many related incidents occurring

- Away from weak actions such as informing staff, training staff and updating policies, towards stronger actions of improving systems and processes

- Away from long lists of actions, towards smaller numbers of high-impact actions

Less attention has been given in Northern Ireland to adverse incidents that do not meet the definition of a Serious Adverse Incident. They are reported, analysed and acted upon at Trust level. Only exceptionally are they considered centrally. The numbers are much greater so the logistics of analysing more would be considerable. However, there is much to be learned from situations when something went wrong in a patient's care but they did not die or suffer serious harm. 


\subsection{THE BENEFITS AND CHALLENGES OF BEING OPEN}

The health and social care system aspires to a 'no blame' culture, or a 'just' culture, in which staff can be open without fear of inappropriate reprisal. In reality, this is not the culture that currently exists. This is not primarily the fault of those delivering health and social care.

Openness is not something that can simply be demanded. It needs the right conditions in order to flourish. The enemy of openness is fear.

When something goes wrong, many patients' and families' first reaction is to want to know who is to blame. The situation often escalates, with the media coverage and political pressure that the detail of the story generates. In an ideal world, leaders of the system should be able to step in to paint a proper picture of the background to these complex events, and to build public understanding that few are a simple case of incompetence and carelessness. Instead, to remove the heat from the situation, approaches are announced that may not be the most effective way to achieve learning. On top of this, day-by-day the media portrays health and social care in a mainly negative light. There has been one inquiry after another. These are conditions conducive to blame and fear, not to transparency and openness.

Despite these adverse conditions, the Review Team found front-line staff willing to talk about problems, and to be open with families and patients when things go wrong. There is a willingness to be open - but there is blame, and there is fear.

Northern Ireland needs to increase the degree of openness and transparency in talking about harm, and decrease the degree of blame and fear. The responsibility cannot lie solely within the health and social care system. They are complex cycles.

\section{Figure 7. The vicious cycle of suspicion and fear}

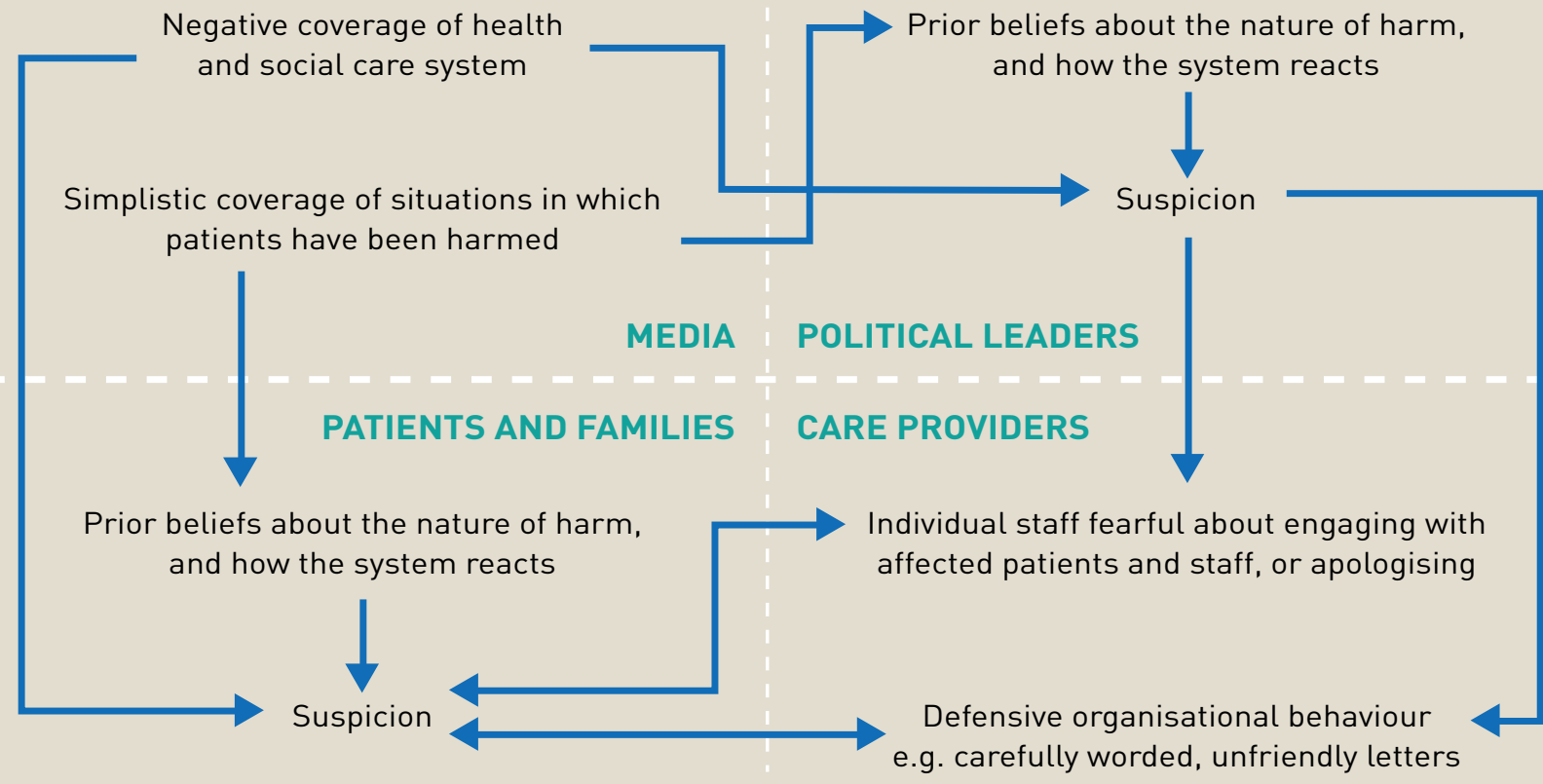


Openness and transparency, blame and fear: these are multi-dimensional issues that cannot be improved directly by legislation, rules or procedures alone. As this Report has made clear, Northern Ireland is far from unique.

\subsubsection{Governance arrangements to promote openness}

Promoting openness and avoiding fear is about culture. Responsibility for this sits with many people, within and beyond the health and social care system. Governance may sound like a blunt tool and, used alone, it would be. But alongside other approaches, appropriate governance arrangements can promote openness and dispel fear.

The Serious Adverse Incident process currently requires Trusts to inform affected patients (or families) that their care is the subject of investigation. In general, they are invited to provide input and are provided with a copy of the investigation report. A checklist has been introduced to prompt investigators to take these steps. This is commendable, and represents a basic, but important, degree of openness with patients and families.

The nature of the involvement with patients and families in the aftermath of a Serious Adverse Incident cannot be shaped by a checklist alone. The Review Team heard from each of the Trusts how they handled this aspect of the policy. It is clear that this is a difficult area to get right. Early contact with the family in the event of a death is important but could come at a time when funeral arrangements are being made and perceived as intrusive or insensitive. The bureaucracy of the procedure can create an official feeling that opens up distance in the relationship with the family. It is important that staff in the Trust have the skill, experience and credibility to communicate with a family. It is helpful to have staff who deal with this situation regularly and have good inter-personal and counselling skills. They should be there with the clinical staff who may encounter the situation less frequently. Experience from elsewhere suggests that regular contact with the patient and family is important, not just a couple of oneoff meetings with long silences in between. In the best services, the patient and family are fully involved in the process of learning and actionplanning. Where this happens, it is empowering for everyone. This is only happening to a limited extent in Northern Ireland currently.

The Serious Adverse Incident process is also overseen by a Designated Review Officer within the Public Health Agency. This is also a welcome feature of the system although there is potential for these officers, or their function, to play a more substantial role.

Every Trust has appropriate arrangements for Serious Adverse Incidents to be discussed within the departments affected. The fact that these conversations are taking place usefully promotes a culture in which talking about harm becomes easier, and openness becomes the norm.

Every Trust also has arrangements for organisation-level oversight of this process. In most, this responsibility sits with a sub-committee of the Trust board. This too is good practice.

When something goes wrong, there is a tendency for the Department of Health, Social Services and Public Safety to deal directly with the Trust's Executive Team, bypassing the board. This happens partly from expediency - because the executive directors are present full-time, and are therefore available to take an urgent phone call from an official concerned about briefing the minister. But it serves to diminish the role of the board, and misses opportunities to build the board's familiarity with these issues and capability in dealing with them.

There is great concern and depth of feeling amongst staff in the system who have attempted to uncover poor standards of 
care and been denigrated. Their role as whistleblowers has placed them in an even more isolated position. This unsatisfactory situation needs to be resolved.

\subsubsection{Perceptions of openness}

The Serious Adverse Incident guidelines include some requirements intended to help openness and transparency. A recent look-back exercise, quality controlled by the Regulation and Quality Improvement Authority, suggests that patients and families are being appropriately informed when a Serious Adverse Incident occurs. This creates a substantially higher degree of openness than is the case in many countries worldwide. In the main, the Trust staff who are leading the investigation are willing to spend time meeting with patients and families.

However, several features of the investigation process too often give patients and families an adverse impression:

- The investigation process is frequently delayed beyond the stipulated timeline, and patients and families experience delays in getting responses to calls and emails. Such delays make people start to wonder, "what is going on?"

- When the investigation process starts, the degree of openness and transparency that the patient and/or family feel they are seeing is highly dependent on the communication skills of the Trust staff that they meet with. Some staff are highly skilled in these potentially difficult meetings; others are not.

- Standard practice is for patients and families to meet with the manager and/or clinician leading the investigation, and not to be asked whom else they would like to meet with. Many, for example, would find it helpful to meet with the staff directly involved in the incident, to put their questions directly, but this is not routinely offered. Such meetings have the potential to be intensely difficult; to be very useful if they go well, but harmful if they go badly.

\subsubsection{Duty of candour}

In 2003, the head of the Review Team las Chief Medical Officer for England) issued a consultation paper, Making Amends, which set out proposals for reforming the approach to clinical negligence in the NHS. One key recommendation was that a duty of candour should be introduced.

As long ago as 1987 Sir John Donaldson Ino relation), who was then Master of the Rolls, said "I personally think that in professional negligence cases, and in particular in medical negligence cases, there is a duty of candour resting on the professional man". There was, at the time of the Making Amends report, no binding decision of the courts on whether such a duty exists.

In November 2014, the General Medical Council and the Nursing \& Midwifery Council issued a joint consultation document proposing the introduction of a professional duty of candour. Such a duty will give statutory force to the General Medical Council's Code of Good Medical Practice for doctors.

In the concomitant healthcare organisational measures introduced in England, a new "Duty of Candour" scheme will mean that hospitals are required to disclose information about incidents that caused harm to patients, and to provide an apology.

In Northern Ireland, it is already a requirement to disclose to patients if their care has been the subject of a Serious Adverse Incident report. There is no similar requirement for adverse incidents that do not cause the more severe degrees of harm. In promoting a culture of openness, there would be considerable advantages in Northern Ireland taking a lead and introducing an organisational duty of candour to match the duty that doctors and nurses are likely to come under from their professional regulators. 


\subsection{THE VOICES OF PATIENTS, CLIENTS AND FAMILIES ARE TOO MUTED}

The best services in the world today give major priority to involving patients and families across the whole range of their activities, from boardlevel policy making, to design of care processes, to quality improvement efforts, to evaluation of services, to working on reducing risk to patients as part of patient safety programmes.

At the heart of the traditional approach to assessing whether a service is responsive to its patients and the public are surveys of patient experience and attitudes. This is still a very important part of modern health and social care. In many major centres whose services are highly rated, such surveys are regularly carried out and used to judge performance at the organisational, service and individual practitioner level, as well as, in some cases, being linked to financial incentives. Indeed, in the United States system, observers say that it was not until surveys of patient experience were linked to dollars that it was taken seriously. This is not a prominent feature of the Northern Ireland system, although there is some very good practice, for example the 10,000 Voices initiative, which has so far drawn on the experience of over 6,000 patients and led to new pathways of care in pain management, caring for children in Emergency Departments, and generally focusing on the areas of dignity and respect.

Looked at from first principles, the kind of questions a user, or potential user, of a service could legitimately require an answer to would include:

How quickly will I first be seen, how quickly will I get a diagnosis and how quickly will I receive definitive treatment?

If my condition is potentially life-threatening, will the local service give me the best odds of survival or could I do better elsewhere?
Will each member of staff I encounter be competent and up-to-date in treating my condition and how will I know that they are?

Does the service have a low level of complications for treatment like mine compared to other services?

How likely am I to be harmed by the care that I receive and what measures does the service take to prevent it?

If I am unhappy with a care-provider's response to a complaint about my care, will the substance of it be looked at by people who are genuinely independent?

Which particular service elsewhere in the United Kingdom, and other parts of the world, achieves the best outcome for someone like me with my condition? How close will my outcome be to that gold standard?

Very few of these questions could be answered reliably in Northern Ireland and other parts of the United Kingdom.

There are many potential themes for patient and family engagement in health and social care, for example:

- in shaping and designing services

- in measuring the quality of care

- in setting standards for consultation

- in shared decision-making

- in self-care of chronic diseases

- in preventing harm

- in giving feedback on practitioner performance

Few services do all of these, some only scratch the surface of genuine involvement, others do a few well. Overall, the Northern Ireland care system is engaged in some of these areas but certainly not in an organised and coherent way. 
The terms of reference of the Review put particlar emphasis on harm. Globally, there is a spectrum in how well health and social care systems interact with patients, clients and families when things go wrong (figure 8). The ideal approach is to engage patients and families completely in the process of learning They often find this hugely beneficial, because it allows them to play an active part in reducing the risk for future patients. It is also immensely powerful for staff, to hear patients' stories firsthand and to work with them to improve things.

\section{Figure 8. Levels of engagement with patients and families when something goes wrong}

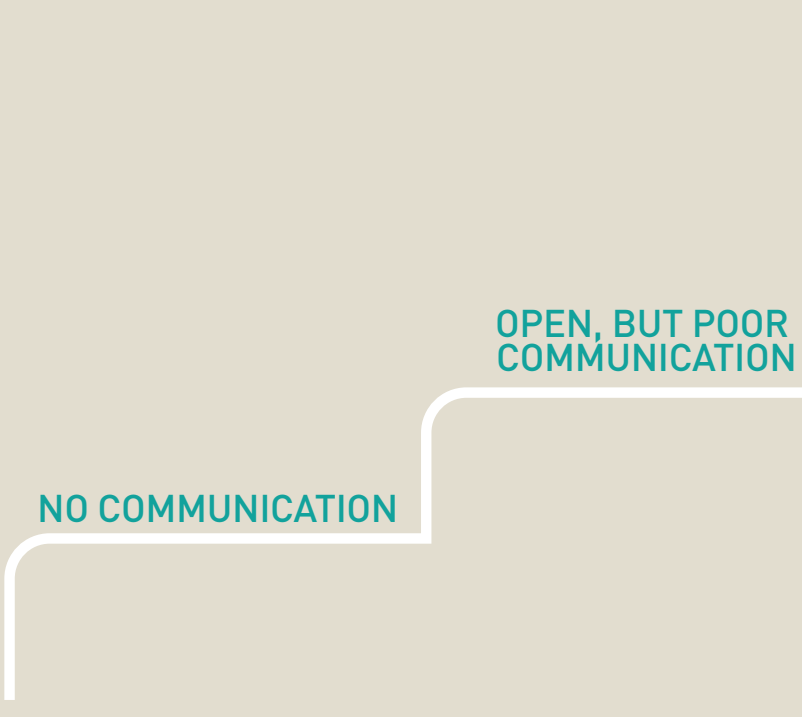

Northern Ireland should aim for level three as an absolute minimum, but strive for level four.

The system is too often falling down to level two because:

- Staff who communicate with patients and families during the Serious Adverse Incident investigation process have variable communication skills - some are excellent, but some are less good. Little formal effort has been made to train staff to manage these difficult interactions well.

- Patients and families are often not offered the opportunity to meet with those who they would like to - the staff directly involved in the incident. Instead, they tend to meet with managers, and with clinicians who were not involved.

- There are frequently delays in the process of investigating a Serious Adverse Incident.

- Patients and families are too often sent letters filled with technical jargon and legalese.

When something goes wrong, the harm itself is intensely difficult for patients and families. Poor communication compounds this enormously. 


\section{CONCLUSIONS}

\subsection{RELATIVE SAFETY OF THE NORTHERN IRELAND CARE SYSTEM}

5.1.1 There is some perception amongst politicians, the press and the public that

Northern Ireland's health and social care system:

- Has fundamental safety problems that are not seen elsewhere

- Is less safe than other parts of the United Kingdom, or comparable countries

- Suffers from lack of transparency, a tendency to cover-up, and an adverse culture more broadly.

5.1.2 The Review found no evidence of deepseated problems of this kind. Northern Ireland is likely to be no more or less safe than any other part of the United Kingdom, or indeed any comparable country globally.

5.1.3 This does not mean that safety can be disregarded, because it is clear from reading the incident reports and accounts of patients' experience that people are being harmed by unsafe care in Northern Ireland, as they are elsewhere. Northern Ireland, like every modern health and social care system, must do all it can to make its patients and clients safer.

\subsection{PROBLEMS GENERATED BY THE DESIGN OF THE HEALTH AND SOCIAL CARE SYSTEM}

5.2.1 There are longstanding, structural elements of the Northern Ireland care system that fundamentally damage its quality and safety. The present configuration of health facilities serving rural and semi-rural populations in Northern Ireland is not fit for purpose and those who resist change or campaign for the status quo are perpetuating an ossified model of care that acts against the interests of patients and denies many 21 st Century standards of care. Many acutely-ill patients in Northern Ireland do not get the same standard of care on a Sunday at $4 \mathrm{am}$ as they would receive on a Wednesday at $4 \mathrm{pm}$ and, therefore, a two-tier service is operating. It may be that local politics means that there is no hope of more modern care for future patients and if so this is a very sad position.

5.2.2 The design of a system to provide comprehensive, high quality, safe, care to a relatively small population like Northern Ireland's needs much more careful thought. This applies to almost all aspects of design including: the role of commissioning, the structuring of provision, the relationship between primary, secondary and social care, the distribution of facilities geographically, the funding flows, the place of regulation, the monitoring of performance, and the use of incentives. Nowhere is the old adage: "I would not start from here" truer than in the Northern Ireland care system today. 
5.2.3 There is widespread uncertainty about who is in overall charge of the system in Northern Ireland. In statutory terms, the Permanent Secretary in the Department of Health, Social Services and Public Safety is chief executive of the health and social care system but how this role is delivered from a policy-making position is not widely understood or visible enough.

5.2.4 In the specific domain of quality and safety itself, whilst it is reflected in the goals and activities of boards and senior management teams in Northern Ireland, it is not yet fully embedded with the commitment and purpose to make a real difference. The Review was most impressed with the work of the South Eastern Trust in this regard. The Review Team could not assess each Trust in depth, but its judgment on the South Eastern Trust is backed up, for example, by the national survey of trainee doctors.

\subsection{FOCUS ON QUALITY AND SAFETY IMPROVEMENT}

5.3.1 Quality 2020 is a ten-year strategy with a bold vision - that the health and social care system should "be recognised internationally, but especially by the people of Northern Ireland, as a leader for excellence in health and social care". Three years on, there is good evidence of the strategy being implemented. An influential steering group oversees the work.

5.3.2 The Review Team judged that Quality 2020 represents a strong set of objectives, and that there is clear evidence of extensive work and of some successes in implementation. However, this does not amount to quality and safety improvement being given the primacy of focus that it needs, and Northern Ireland is not seeing the wood for the trees about the need to establish crucial aspects of quality and safety improvement which are not well represented at present: clinical leadership, cultural change, data linked to goals, and standardisation. 


\subsection{THE EXTENT TO WHICH SERIOUS ADVERSE INCIDENT REPORTING IMPROVES SAFETY}

5.4.1 The system of Serious Adverse Incident reporting in Northern Ireland has been an important way to ensure that the most severe forms of harm that are inadvertently caused by care processes are recognised and investigated.

5.4.2 The Serious Adverse Incident process fulfils five main purposes:

- a public accountability function

- a response to the patients and families involved

- a communications alert route

- a barometer of risk within health and social care

- a foundation for learning and improvement

5.4.3 The kinds of incidents reported into this system appear little different to other parts of the United Kingdom and are similar to many other parts of Europe, North America and Australasia. Many harmful events are potentially avoidable and the human cost to patients and families in Northern Ireland is of grave concern, as it is in other jurisdictions.

5.4.4 Good practice elsewhere in the world suggests that patients who suffer harm and their families should be fully informed about what has happened, how it happened and what will be done to prevent another similar occurrence. More than this, they should be fully engaged in working with the organisation to make change. Patient and family engagement is a good and established feature of Serious Adverse Incident reporting in Northern Ireland but it often falls short of this fully engaged scenario. The extent to which it is valued and trusted by patients and families appears to vary, depending on the staff communicating with them.
5.4.5 The design for the specification, and recording, of information on each Serious Adverse Incident is sub-optimal particularly in gathering appropriate information on causation; this hinders aggregation of data to monitor trends and assess the impact of interventions.

5.4.6 The process for investigating Serious Adverse Incidents is clearly set out and involves root cause analysis-type methods. In many cases, it lacks sufficient depth in key areas such as human factors analysis. The degree of oversight by supervisory officials (the Designated Review Officers) is variable in extent and timeliness. Local health and social care staff generally approach the task of investigation conscientiously but many lack the training and experience to reach a standard of international best practice in unequivocally identifying the cause and specifying the actionable learning. They get little expert help and guidance in undertaking this activity.

5.4.7 The most important test of the capability of a patient safety incident reporting system is its effectiveness in reducing future harm of the kind that is being reported to it. Unfortunately, there are few places around the world where there is a powerful flow of learning that moves from identifying instances of avoidable harm, through understanding why they did or could happen, to successful elimination of the risk for future patients. Northern Ireland is no exception to this regrettable state of affairs.

5.4.8 There are two main levels of learning from Serious Adverse Incidents in Northern Ireland. The first is local. The lack of a consistently high standard of investigation and actionplanning are barriers to effective risk-reduction within health and social care organisations. Another barrier is the limited degree to which front-line staff are involved in discussing and seeking solutions to things that have gone wrong. Experience elsewhere suggests that this practical and intellectual engagement, 
if well-led, often sparks great interest and commitment to patient safety amongst frontline staff. This is not really happening in Northern Ireland at present, for a number of reasons. Firstly, staff do not have the time and space to do it and the leadership of Trusts is not consistently creating and facilitating such opportunities. The Regulation and Quality Improvement Authority has established training in Root Cause Analysis for front-line staff, and this will help. Secondly, the specified rules of the Serious Adverse Incident system mean that Trusts are under a great deal of pressure to meet the time-scales laid down and are often dealing with many such cases simultaneously. As a result, the activity is too often slipping into an incident management role or worse a necessary chore that 'feeds the beast'.

\subsubsection{The second level of learning is across} the Northern Ireland health and social care system as a whole. The main role is played by the Health and Social Care Board working with the Public Health Agency land the Regulation and Quality Improvement Authority where appropriate). These bodies have established a multi-disciplinary Quality Safety and Experience Group that undertakes much of the work in assessing patterns, trends and concerns arising from the analysis of locally-generated Serious Adverse Incidents and deciding what action needs to be taken on a Northern Irelandwide basis. It does so by issuing learning letters, reports, guidance, newsletters and other specified action that the service needs to take. This is a valuable function from which considerable action aimed at improvement has flowed. Experience of improving patient safety elsewhere has shown that specifying action on a particular safety problem is not the same thing as implementing the change required. The latter is often much more difficult and depends on factors such as the systems, culture, attitudes, local priorities and leadership in the organisation receiving the action note. In the Northern Ireland care system more skill needs to be added to the implementation process. This is closely linked to the difficulties that arise when local services feel overloaded with central guidance and requirements for action. They only have enough management and clinical leadership capacity to implement a small number of changes at a time.

5.4.10 General practitioners, and others in primary care, report their Serious Adverse Incidents directly to the Health and Social Care Board, not through any of the Trusts. Levels of reporting of patient safety incidents in primary care services around the world are very low and much less is known about the kinds of harm that arise in this setting compared to hospitals. It is not surprising that the same is so in Northern Ireland. Another aspect of the primary care dimension is that many of the incidents that the Review discussed with the Trusts in Northern Ireland had a primary care element in the key areas of the care processes that had failed, yet general practitioners seemed to be less frequently involved in the investigation and planning of remedial action.

5.4.11 There are two particular aspects of the criteria for Serious Adverse Incident reporting in Northern Ireland that are not working in the best interests of a successful system. Firstly, the requirement that every death of a child in receipt of health and social care should automatically become a Serious Adverse Incident is causing major problems. A proportion of such deaths every month are due to natural causes. Some of the conditions concerned - for example, terminal cancer and serious congenital abnormalities - are particularly harrowing for the parents. After the death of a child, in such circumstances, for a family to be told that their child's death has been categorised as a Serious Adverse Incident carries the clear implication that the quality or safety of care was poor and at fault or even that the death could have been avoided. This can be enormously distressing for families and 
is grueling for staff. It is cruel, unnecessary and liable to undermine public confidence in children's services.

5.4.12 Secondly, using the same time-scales for investigating Serious Adverse Incidents in mental health as in in other fields of care is also causing major problems. The complexity of many mental health cases, the long past history of many such patients and clients, and the number of people and organisations who may be able to contribute relevant information to the investigation mean that a longer period is necessarily required to get to the truth than is currently permitted.

5.4.13 Overall, the system of Serious Adverse Incident reporting in Northern Ireland, in comparison to best practice, scores highly on securing accountability, reasonably highly on the level of reporting, does moderately well on meaningful engagement with patients and families, and is weak in producing effective, sustained reduction in risk. Also, the climate of accountability and intense political and media scrutiny does not sit easily with what best practice has repeatedly shown is the key to making care safer: a climate of learning not judgment.

5.4.14 The Review concluded that front-line clinical staff are insufficiently supported to fulfill the role of assessing and improving the quality and safety of the care that they and their teams provide. The lack of time, the paucity of reliable, well-presented data, the absence of in-service training in quality improvement methods, and the patchiness of clinical leadership are all major barriers to achieving this vital shift to mass clinical engagement.

\subsection{OPENNESS WITH PATIENTS AND FAMILIES}

5.5.1 The Serious Adverse Incident investigation system contains, in the view of the Review Team, sufficient checks and balances to ensure that affected patients and families are informed that something went wrong, except in exceptional circumstances.

5.5.2 Such mechanisms are part of good governance, but alone are insufficient. It will be culture - not accountability - that increases the reporting of harm, and staff's comfort in talking openly about harm.

5.5.3 Those conducting investigations are committed to rigorous investigation, and to being open with patients and families about what is found. But whilst some communicate well in person and in writing, others are less strong. This can come across to families as a lack of openness.

5.5.4 High-profile inquiries and negative media coverage have led some to believe that there is widespread cover-up of harm in the health and social care system. This is simply inconsistent with what the Review Team observed, which was a system trying, as many others in the world are, to get to grips with the difficult problem of patient safety.

5.5.5 Fear and suspicion powerfully inhibit openness. The health and social care system needs to rise to the challenge of tackling these threats head on. Perception is important - even simple delays and communication weaknesses can fuel suspicion. And if staff hear more from the media than direct from their leaders, this does not dispel fear. 
Recommendation 1:

Coming together for world-class care

A proportion of poor quality, unsafe care occurs because local hospital facilities in some parts of Northern Ireland cannot provide the level and standard of care required to meet patients needs 24 hours a day, 7 days a week. Proposals to close local hospitals tend to be met with public outrage, but this would be turned on its head if it were properly explained that people were trading a degree of geographical inconvenience against life and death. Finding a solution should be above political self-interest.

We recommend that all political parties and the public accept in advance the recommendations of an impartial international panel of experts who should be commissioned to deliver to the Northern Ireland population the configuration of health and social care services commensurate with ensuring world-class standards of care.

\section{Recommendation 2:}

\section{Strengthened commissioning}

The provision of health and social care in Northern Ireland is planned and funded through a process of commissioning that is currently tightly centrally-controlled and based on a crude method of resource allocation. This seems to have evolved without proper thought as to what would be most effective and efficient for a population as small as Northern Ireland's. Although commissioning may seem like a behind-the-scenes management black box that the public do not need to know about, quality of the commissioning process is a major determinant of the quality of care that people ultimately receive.

We recommend that the commissioning system in Northern Ireland should be redesigned to make it simpler and more capable of reshaping services for the future. A choice must be made to adopt a more sophisticated tariff system, or to change the funding flow model altogether.

\section{Recommendation 3:}

Transforming Your Care - action not words

The demands on hospital services in Northern Ireland are excessive and not sustainable. This is a phenomenon that is occurring in other parts of the United Kingdom. Although triggered by multiple factors, much of it has to do with the increasing levels of frailty and multiple chronic diseases amongst older people together with too many people using the hospital emergency department as their first port of call for minor illness. High-pressure hospital environments are dangerous to patients and highly stressful for staff. The policy document Transforming Your Care contains many of the right ideas for developing high quality alternatives to hospital care but few believe it will ever be implemented or that the necessary funding will flow to it. Damaging cynicism is becoming widespread.

We recommend that a new costed, timetabled implementation plan for Transforming Your Care should be produced quickly. We further recommend that two projects with the potential to reduce the demand on hospital beds should be launched immediately: the first, to create a greatly expanded role for pharmacists; the second, to expand the role of paramedics in pre-hospital care. Good work has already taken place in these areas and more is planned, but both offer substantial untapped potential, particularly if front-line creativity can be harnessed. We hope that the initiatives would have high-level leadership to ensure that all elements of the system play their part. 
Recommendation 4:

\section{Self-management of chronic disease}

Many people in Northern Ireland are spending years of their lives with one or more chronic diseases. How these are managed determines how long they will live, whether they will continue to work, what disabling complications they will develop, and the quality of their life. Too many such people are passive recipients of care. They are defined by their illness and not as people. Priority tends to go to some diseases, like cancer and diabetes, and not to others where provision remains inadequate and fragmented. Quality of care, outcome and patient experience vary greatly. Initiatives elsewhere show that if people are given the skills to manage their own condition they are empowered, feel in control and make much more effective use of services.

We recommend that a programme should be established to give people with long-term illnesses the skills to manage their own conditions. The programme should be properly organised with a small full-time coordinating staff. It should develop metrics to ensure that quality, outcomes and experience are properly monitored. It should be piloted in one disease area to begin with. It should be overseen by the Long Term Conditions Alliance.

\section{Recommendation 5: Better regulation}

The regulation of care is a very important part of assuring standards, quality and safety in many other jurisdictions. For example, the Care Quality Commission has a very prominent role in the inspection and registration of healthcare providers in England. In the USA, the Joint Commission's role in accreditation means that no hospital wants to fall below the standards set or it will lose reputation and patients. The Review Team was puzzled that the regulator in Northern Ireland, the Regulation and Quality Improvement Authority, was not mentioned spontaneously in most of the discussions with other groups and organisations. The Authority has a greater role in social care than in health care. It does not register, or really regulate, the Trusts that provide the majority of healthcare and a lot of social care. This lighttouch role seems very out of keeping with the positioning of health regulators elsewhere that play a much wider role and help support public accountability. The Minister for Health, Social Services and Patient Safety has already asked that the regulator start unannounced inspections of acute hospitals from 2015, but these plans are relatively limited in extent.

We recommend that the regulatory function is more fully developed on the healthcare side of services in Northern Ireland. Routine inspections, some unannounced, should take place focusing on the areas of patient safety, clinical effectiveness, patient experience, clinical governance arrangements, and leadership. We suggest that extending the role of the Regulation and Quality Improvement Authority is tested against the option of outsourcing this function (for example, to Healthcare Improvement Scotland, the Scottish regulator). The latter option would take account of the relatively small size of Northern Ireland and bring in good opportunities for benchmarking. We further recommend that the Regulation and Quality Improvement Authority should review the current policy on whistleblowing and provide advice to the Minister. 


\section{Recommendation 6:}

\section{Making incident reports really count}

The system of incident reporting within health and social care in Northern Ireland is an important element of the framework for assuring and improving the safety of care of patients and clients. The way in which it works is falling well below its potential for the many reasons explained in this report. Most importantly, the scale of successful reduction of risk flowing from analysis and investigation of incidents is too small.

We recommend that the system of Serious Adverse Incident and Adverse Incident reporting should be retained with the following modifications:

- deaths of children from natural causes should not be classified as Serious Adverse Incidents;

- there should be consultation with those working in the mental health field to make sensible changes to the rules and timescales for investigating incidents involving the care of mental health patients;

- a clear policy and some re-shaping of the system of Adverse Incident reporting should be introduced so that the lessons emanating from cases of less serious harm can be used for systemic strengthening Ithe Review Team strongly warns against uncritical adoption of the National Reporting and Learning System for England and Wales that has serious weaknesses);

- a duty of candour should be introduced in Northern Ireland consistent with similar action in other parts of the United Kingdom;

- a limited list of Never Events should be created

- a portal for patients to make incident reports should be created and publicised

- other proposed modifications and developments should be considered in the context of Recommendation 7.

\section{Recommendation 7:}

A beacon of excellence in patient safety

There is currently a complex interweaving of responsibilities for patient safety amongst the central bodies responsible for the health and social care system in Northern Ireland. The Department of Health, Social Services and Public Safety, the Health and Social Care Board, and the Regulation and Quality Improvement Authority all play a part in: receiving Serious Adverse Incident Reports, analysing them, over-riding local judgments on designation of incidents, requiring and overseeing investigation, auditing action, summarising learning, monitoring progress, issuing alerts, summoning-in outside experts, establishing inquiries, checking-up on implementation of inquiry reports, declaring priorities for action, and various other functions. The respective roles of the Health and Social Care Board and the Public Health Agency are clearly specified in legal regulations but seem very odd to the outsider. The Health and Social Care Board has no full-time officers of its own who lead on quality and safety and no in-house medical or nursing director. These functions are grafted on from the Public Health Agency. The individuals concerned have done some excellent work on quality and patient safety and carry out their roles very conscientiously. However, symbolically, and on grounds of organisational coherence, it appears strange that the main body responsible for planning and securing care does not hold these functions in the heart of its business. The Department of Health, Social Services and Public Safety's role on paper is limited to policy-making but, in practice, steps in regularly on various aspects of quality and safety. The Review Team thought long and hard before making a recommendation in this area. In the end, we believe action is imperative for two reasons: firstly, the present central arrangements are byzantine and confusing; secondly, the overwhelming need is for development of the present system to make it much more successful in bringing about improvement. Currently, almost all the activities 
(including those listed above) are orientated to performance management not development. There is a big space for a creative, positive and enhancing role.

We recommend the establishment of a Northern Ireland Institute for Patient Safety, whose functions would include:

- carrying out analyses of reported incidents, in aggregate, to identify systemic weaknesses and scope for improvement;

- improving the reporting process to address under-reporting and introducing modern technology to make it easier for staff to report, and to facilitate analysis;

- instigating periodic audits of Serious Adverse Incidents to ensure that all appropriate cases are being referred to the Coroner;

- facilitating the investigation of Serious Adverse Incidents to enhance understanding of their causation;

- bringing wider scientific disciplines such as human factors, design and technology into the formulation of solutions $b$ problems identified through analysis of incidents;

- developing valid metrics to monitor progress and compare performance in patient safety;

- analysing adverse incidents on a sampling basis to enhance learning from less severe events;

- giving front-line staff skills in recognising sources of unsafe care and the improvement tools to reduce risks;

- fully engaging with patients and amilies to involve them as champions in the Northern Ireland patient safety program, including curating a library of patient stories for use in educational and staff induction programmes;

- creating a cadre of leaders in patient safety across the whole health and social care system;

- initiating a major programme to build safety resilience into the health and social care system.

\section{Recommendation 8:}

System-wide data and goals

The Northern Ireland Health and Social Care system has no consistent method for the regular assessment of its performance on quality and safety at regional-level, Trust-level, clinical service-level, and individual doctorlevel. This is in contrast to the best systems in the world. The Review Team is familiar with the Cleveland Clinic. That service operates by managing and rewarding performance based on clinically-relevant metrics covering areas of safety, quality and patient experience. This is strongly linked to standard pathways of care where outcome is variable or where there are high risks in a process.

We recommend the establishment of a small number of systems metrics that can be aggregated and disaggregated from the regional level down to individual serviœ level for the Northern Ireland health and social care system. The measures should be those used in validated programmes in North America (where there is a much longer tradition of doing this) so that regular benchmarking can take place. We further recommend that a clinical leadership academy is established in Northern Ireland and that all clinicl staff pass through it. 


\section{Recommendation 9:}

Moving to the forefront of new technology

The potential for information and digital technology to revolutionise healthcare is enormous. Its impact on some of the longstanding quality and safety problems of health systems around the world is already becoming evident in leading edge organisations. These developments include: the electronic medical record, electronic prescribing systems for medication, automated monitoring of acutelyill patients, robotic surgery, smartphone applications to manage workload in hospitals at night, near-patient diagnostics in primary care, simulation training, incident reporting and analysis on mobile devices, extraction of real-time information to assess and monitor service performance, advanced telemedicine, and even smart kitchens and talking walls in dwellings adapted for people with dementia. There is no organised approach to seeking out and making maximum use of technology in the Northern Ireland care system. It could make a big difference in resolving some of the problems described in this report. There is evidence of individual Trusts making their own way forward on some technological fronts, but this uncoordinated development is inappropriate the size of Northern Ireland is such that there should be one clear, unified approach.
We recommend that a small Technology Hub is established to identify the best technological innovations that are enhancing the quality and safety of care around the world and to make proposals for adoption in Northern Ireland. It is important that this idea is developed carefully. The Technology Hub should not deal primarily with hardware and software companies that are selling products. The emphasis should be on identifying technologies that are in established use, delivering proven benefits, and are highly valued by management and clinical staff in the organisations concerned. They should be replicable at Northern Ireland-scale. The overall aim of this recommendation is to put the Northern Ireland health and social care system in a position where it has the best technology and innovation from all corners of the world and is recognised as the most advanced in Europe. 
Recommendation 10:

\section{A much stronger patient voice}

In the last decade, policy-makers in health and social care systems around the world have given increasing emphasis to the role of patients and family members in the wider aspects of planning and delivering services. External reviews - such as the Berwick Report in England - have expressed concern that patients and families are not empowered in the system. Various approaches have been taken worldwide to address concerns like these. Sometimes this has been through system features such as choice and personally-held budgets, sometimes through greater engagement in fields like incident investigation, sometimes through user experience surveys and focus groups, and sometimes through direct involvement in the governance structures of institutions. In the USA, patient experience data now forms part of the way that hospitals are paid and in some it determines part of the remuneration of individuals. This change catalysed the centrality of patients to the healthcare system in swathes of North America. Observers say that the big difference was when dollars were linked to the voice of patients. Northern Ireland has done some good work in the field of patient engagement, in particular the requirement to involve patients and families in Serious Adverse Incident investigation, the 10,000 voices initiative, in the field of mental health and in many aspects of social care. Looked at in the round, though patients and families have a much weaker voice in shaping the delivery and improvement of care than is the case in the best healthcare systems of the world.

We recommend a number of measures to strengthen the patient voice:

- more independence should be introduced into the complaints process; whilst all efforts should be made to resolve a complaint locally, patients or their families should be able to refer their complaint to an independent service. This would look again at the substance of the complaint, and use its good offices to bring the parties together to seek resolution. The Ombudsman would be the third stage and it is hoped that changes to legislation would allow his reports to be made public;

- the board of the Patients and Client Council should be reconstituted to include a higher proportion of current or former patients or clients of the Northern Ireland health and social care system;

- the Patients and Client Council should have a revised constitution making it more independent;

- the organisations representing patients and clients with chronic diseases in Northern Ireland should be given a more powerful and formal role within the commissioning process, the precise mechanism to be determined by the Department of Health, Social Services and Public Safety;

- one of the validated patient experience surveys used by the Centers for Medicare and Medicaid Services in the USA (with minor modification to the Northern Ireland context) to rate hospitals and allocate resources should be carried out annually in Northern Ireland; the resulting data should be used to improve services, and assess progress. Finally and importantly, the survey results should be used in the funding formula for resource allocation to organisations and as part of the remuneration of staff (the mechanisms to be devised and piloted by the Department of Health, Social Services, and Public Safety).

In implementing the above recommendations, the leaders of the Northern Ireland health and social care system should be clear in their ambition, which is in our view realistic, of making Northern Ireland a world leader in the quality and safety of its care. Northern Ireland is the right place for such a transformation, and now is the right time. 


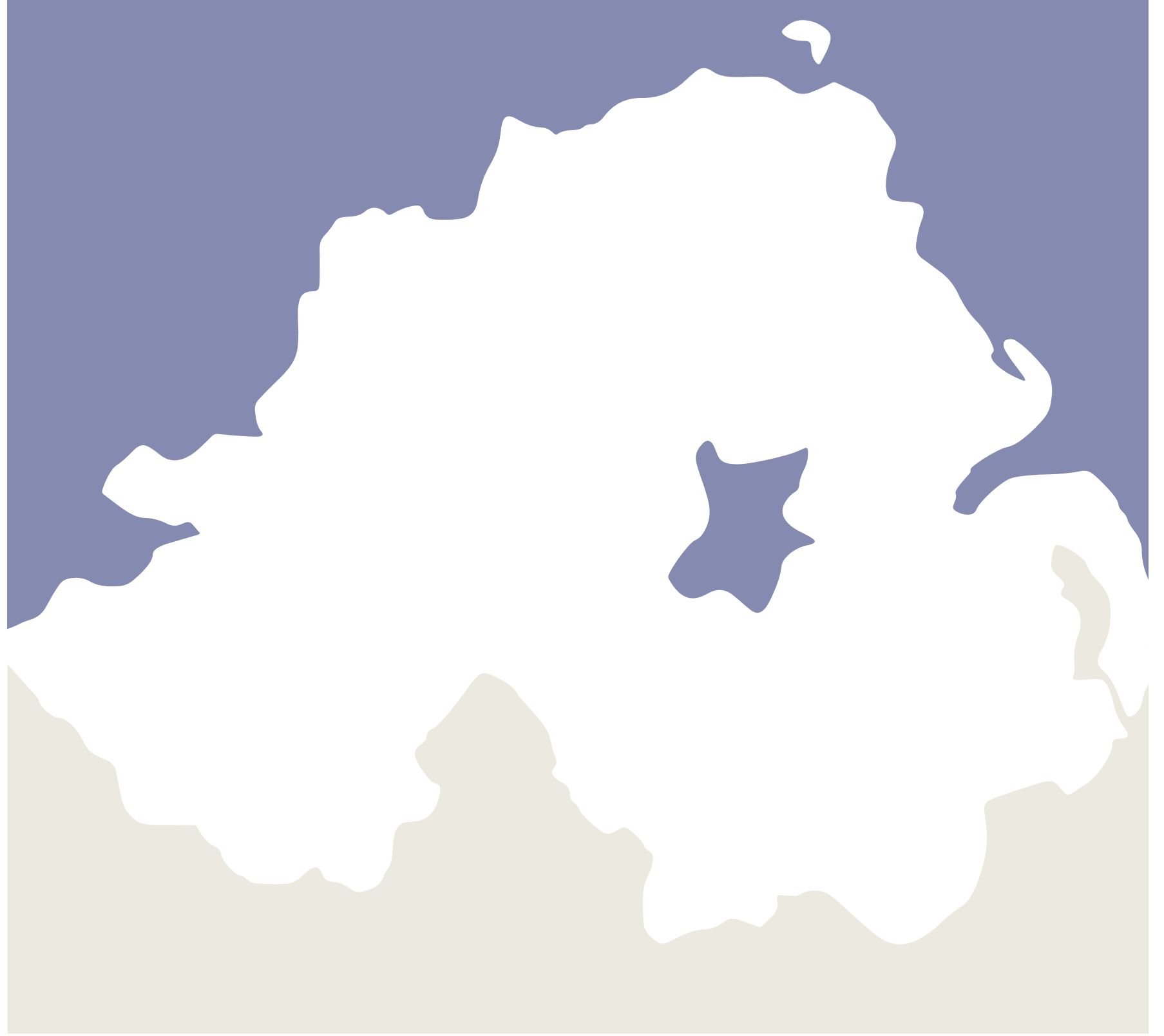

